\title{
Wall loss of atomic nitrogen determined by ionization threshold mass spectrometry
}

M. Sode, ${ }^{1, a)}$ T. Schwarz-Selinger, ${ }^{1}$ W. Jacob, ${ }^{1}$ and H. Kersten ${ }^{2}$

1) Max-Planck-Institut für Plasmaphysik, Boltzmannstraße 2, D-85748 Garching, Germany.

${ }^{2)}$ Institute for Experimental and Applied Physics, ChristianAlbrechts-Universität zu Kiel, Leibnizstraße 11-19, D-24098 Kiel, Germany.

(Dated: 19 November 2014)

In the afterglow of an inductively coupled $\mathrm{N}_{2}$ plasma relative $\mathrm{N}$ atom densities are measured by ionization threshold mass spectrometry (ITMS) as a function of time in order to determine the wall loss time $t_{w \mathrm{~N}}$ from the exponential decay curves. The procedure is performed with two mass spectrometers on different positions in the plasma chamber. $t_{w \mathrm{~N}}$ is determined for various pressures, i.e., for 3.0, 5.0, 7.5, and $10 \mathrm{~Pa}$. For this conditions also the internal plasma parameters electron density $n_{e}$ and electron temperature $T_{e}$ are determined with the Langmuir probe and the rotational temperature $T_{\text {rot }}^{\mathrm{N}_{2}}$ of $\mathrm{N}_{2}$ is determined with the optical emission spectroscopy. For $T_{\text {rot }}^{\mathrm{N}_{2}}$ a procedure is presented to evaluate the spectrum of the transition $v^{6}=0 \rightarrow v^{\prime \prime}=2$ of the second positive system $\left(C^{3} \Pi_{u} \rightarrow B^{3} \Pi_{g}\right)$ of $N_{2}$. With this method a gas temperature of $610 \mathrm{~K}$ is determined. For both mass spectrometers an increase of the wall loss times of atomic nitrogen with increasing pressure is observed. The wall loss time measured with the first mass spectrometer in the radial center of the cylindrical plasma vessel increases linearly from $0.31 \mathrm{~ms}$ for $3 \mathrm{~Pa}$ to $0.82 \mathrm{~ms}$ for $10 \mathrm{~Pa}$. The wall loss time measured with the second mass spectrometer (further away from the discharge) is about 4 times higher. A model is applied to describe the measured $t_{w \mathrm{~N}}$. The main loss mechanism of atomic nitrogen for the considered pressure is diffusion to the wall. The surface loss probability $\beta_{\mathrm{N}}$ of atomic nitrogen on stainless steel was derived from $t_{w \mathrm{~N}}$ and is found to be 1 for the present conditions. The difference in wall loss times measured with the mass spectrometers on different positions in the plasma chamber is attributed to the different diffusion lengths.

PACS numbers: 52.25.-b, 82.33.Xj, 52.40.Hf, 52.70.Nc

Keywords: surface loss probability, ionization threshold mass spectrometry, nitrogen radical, $\mathrm{N}_{2}$ rotational temperature

a) Electronic mail: maik.sode@ipp.mpg.de 


\section{INTRODUCTION}

Plasma process technology plays an important role in a wide range of different industrial applications such as semiconductors, solar cells, and display manufacturing. Reactive plasmas are used for etching, coating and surface treatment. In a large variety of cases, etching or deposition processes are based on the reactivity of the radical particles involved. Therefore, quantifying the radical particle densities is a first and important step for an improved understanding of plasma-surface-interaction processes. One relevant radical is atomic nitrogen, used in semiconductor manufactoring for nitridation, for example, in the fabrication of gate insulators or interlayer dielectric films ${ }^{1,2}$. For low-pressure plasmas atomic nitrogen is mainly produced by electron-induced dissociation of $\mathrm{N}_{2}$ which is predominantly affected by the electron density and electron temperature of the respective plasma. The loss of atomic nitrogen in such plasmas is determined by the flux to the wall ${ }^{3-8}$. In our case, as in most other cases, the wall loss is determined by recombination of atomic nitrogen to form molecular nitrogen which desorbs from the surface. This wall loss of atomic nitrogen is described by the surface loss probability $\beta_{\mathrm{N}}$ which is a material specific property. Kersten et al. ${ }^{9}$ studied the energy flux with a thermal probe in the spacial afterglow of a $\mathrm{N}_{2}$ plasma. They changed the substrate material of the thermal probe under comparable experimental plasma conditions so that the $\mathrm{N}$ influx was the same for all studied substrate materials. They observed that the power influx of the recombining radicals onto the surface of the thermal probe depends sensitively on the material substrate of the thermal probe. This was interpreted by different $\beta_{\mathrm{N}}$ values for the different materials.

$\beta_{\mathrm{N}}$ is not only different for different materials, but even for the same material published values for $\beta_{\mathrm{N}}$ vary between different experiments. Since the recombination process of radicals on surfaces and, hence, $\beta_{\mathrm{N}}$ are a function of the surface temperature, a possible explanation for different $\beta_{\mathrm{N}}$ could be the different surface temperatures. Furthermore it has to be kept in mind that $\beta_{\mathrm{N}}$ values are in general determined from measured data on the basis of a diffusion model which describes the transport of the radical particles to the chamber walls. This evaluation procedure depends to a large extent on assumptions on the relevant geometry of the plasma chamber and on the assumption about the $\mathrm{N}$ atom temperature. In principle, there are several methods to obtain experimental values of $\beta_{\mathrm{N}}$. The first method is to measure the power influx onto a thermal probe in the spacial afterglow as mentioned above (see, e.g., Refs. ${ }^{9-11}$ ). However, for this method the absolute density and temperature of atomic nitrogen $n_{\mathrm{N}}$ has to be known. Another method is to apply a rate equation model with further input parameters, i.e., electron density and electron temperature to derive $\beta_{\mathrm{N}}$ indirectly from the atomic $\mathrm{N}$ density in the steady state plasma, as applied by Singh et al. ${ }^{4}$. However, this indirect method is charged with a larger uncertainty due to error propagation of the uncertainties of the various input parameters. The third method is to measure the decay of the $\mathrm{N}$ atom density in the temporal afterglow of a $\mathrm{N}_{2}$ plasma. With this method $t_{w \mathrm{~N}}$ is measured directly. There exist several spectroscopic methods for detecting the radical density time-resolved with a high time resolution of the order of milliseconds or even microseconds: vacuum ultra violett absorption spectroscopy (VUVAS) ${ }^{5}$, two-photonabsorption laser-induced fluorescence $(\mathrm{TALIF})^{6,7}$, and quantum cascade laser absorption 
spectroscopy (QCLAS) ${ }^{12}$.

In the work presented here, relative $\mathrm{N}$ atom densities were measured in the afterglow of pure $\mathrm{N}_{2}$ plasmas as a function of time by ionization threshold mass spectrometry (ITMS) in order to determine the wall loss time $t_{w \mathrm{~N}}$. The wall loss times were determined for various pressures and from these data the surface loss probability of atomic nitrogen on stainless steel $\beta_{\mathrm{N}}$ was derived. The procedure is performed with two independent mass spectrometers at different positions in the plasma chamber. The first mass spectrometer (plasma monitor, PM) is located in the radial center of the plasma chamber measuring the atomic nitrogen density of the hot plasma zone. The second mass spectrometer (molecularbeam mass spectrometer, MBMS) measures the radical density on the radial side walls of the plasma chamber far away from the hot plasma zone. To our knowledge, measurements of the wall loss time at two different positions in the same plasma chamber were not yet published neither for nitrogen nor for other wall loss times of radical species such as hydrogen or oxygen. In addition, contrary to most spectroscopic determination ${ }^{5-7}$ of the nitrogen radical density where the density is averaged over the line of sight this work measures the local radical density at two very different positions.

\section{EXPERIMENT}

\section{A. Experimental setup}

The experimental setup consists of a cylindrical stainless steel plasma chamber shown schematically in Fig. 1. A detailed description of the experimental set-up can be found in Ref. ${ }^{13}$. In short: The discharge is generated by inductive coupling using a planar coil with 5 turns and $100 \mathrm{~mm}$ in diameter. The coil is driven by a radio frequency $(r f)$ generator operating at $13.56 \mathrm{MHz}$ with a maximum power of 600 Watt (Dressler Cesar 136). The coil is separated from the vacuum chamber by a quartz dome ${ }^{14}$. The top part of the dome is $10 \mathrm{~mm}$ thick and acts as the dielectric window for the rf power. The outer diameter $d_{e l}$ of the quartz dome and the stainless steel electrode which is located opposite the dielectric window is $d_{e l}=2 r_{e l}=131 \mathrm{~mm}$ where $r_{e l}$ is the corresponding radius. The axial distance $l_{e l}$ between the electrode and the quartz window is $60 \mathrm{~mm}$. At $r_{e l}$ there is no radial side wall between the electrode and dielectric window. The stainless steel vacuum chamber has a radius of $r_{c h}=125 \mathrm{~mm}$ and a total height of $l_{c h}=360 \mathrm{~mm}$. For the description of the plasma chamber setup a coordinate system with cylindrical geometry $(r$ - radial component, $l$ - axial component) is used whose origin is placed at the radial center of the plasma chamber $30 \mathrm{~mm}$ below the stainless steel electrode (see also Fig. 1).

The plasma chamber is pumped by a turbo molecular pump with Hohlweck stage. The incoming gas flows are adjusted with mass-flow controllers. The working pressure is measured with a capacitance manometer before plasma ignition. The pressure stays constant within the experimental uncertainty of $2 \%$ of the capacitance manometer when the plasma is ignited.

The electron energy distribution function (EEDF), the electron temperature $T_{e}$, and the electron density $n_{e}$ were determined by a single-tip Langmuir probe system ${ }^{13} . T_{e}$ is calculated 


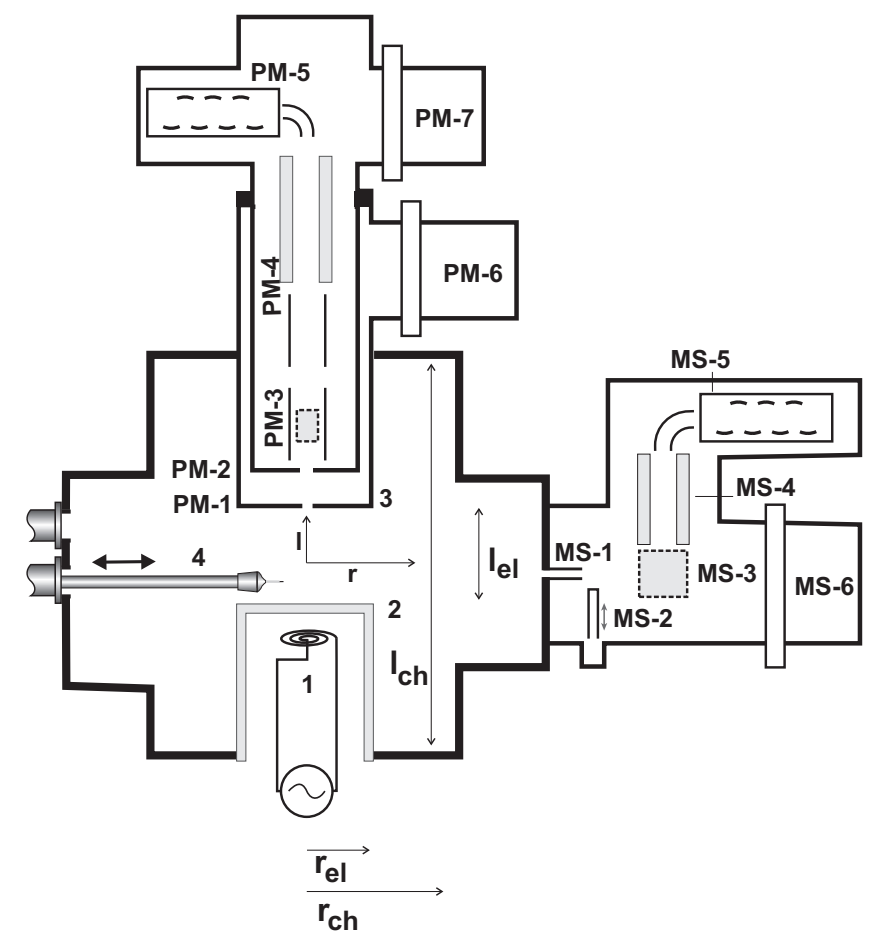

FIG. 1. Schematic representation of the plasma chamber: 1 - planar inductive coil, 2 - quartz dome, 3- stainless steel housing for the PM, 4 - Langmuir probe. Plasma monitor set-up: PM-1 - first orifice $(100 \mu \mathrm{m})$, PM-2 - second orifice $(500 \mu \mathrm{m})$, PM-3 - ion optics and ionizer, PM-4 - energy and mass analyzer, PM-5 - secondary electron multiplier, PM-6 - first pumping stage, PM-7 - second pumping stage. Molecular-beam mass spectrometer set-up: MS-1 - capillary tube, MS-2 - shutter, MS-3 - cross-beam ion source, MS-4 - quadrupol mass filter, MS-5 - secondary electron multiplier, MS-6 - pumping system.

by the potential difference $\Delta V=V_{p l}-V_{f l} \operatorname{method}^{13}$, where $V_{p l}$ and $V_{f l}$ denote the plasma and the floating potential, respectively and $n_{e}$ is calculated by integrating the EEDF ${ }^{13}$. The Langmuir probe measurements were performed in the center of the plasma $(r=0 \mathrm{~mm})$, $20 \mathrm{~mm}$ above the quartz window.

Optical emission spectroscopy (OES) was applied to measure the gas temperature. The used spectrometer was a Czerny-Turner spectrograph (Acton SpectraPro 275) with a focal length of $275 \mathrm{~mm}$. The experiments were conducted with a 1800 lines/mm grating, which has a measured resolution of $0.15 \mathrm{~nm}$ at $\lambda=600 \mathrm{~nm}$. The light was detected by a CCD array (EEV 256x1024 OE CCD30, PIXIS, Princeton Instruments). The measured signal $D(\lambda)$ (in arbitrary units) results from the emitted photons integrated along the line of sight through the plasma as a function of the wavelength $\lambda$ in a time interval $t_{\text {int }}$. The signal was relatively quantified by multiplying with the relative sensitivity curve $R(\lambda)$ obtained for our experimental setting using a calibrated halogen lamp and $D_{2}$ arc discharge light source for calibration $^{8,13}$.

For radicals the density in the afterglow and hence the signal intensity is low. To increase counting statistics repetition of the afterglow state is necessary. To obtain a high repetition rate of the afterglow a pulsed plasma was used. The pulsed plasma is operated with a 
power-on phase $\left(P_{o n}\right)$ with a duration of $t_{\text {on }}$ and a power-off phase $\left(P_{o f f}\right)$ with a duration

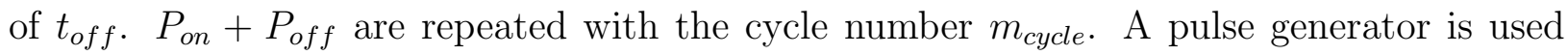
which switches on and off the $r f$ generator and provides the trigger signal for the mass spectrometer measurements. The signal pulses (measuring events) of the secondary electron multipliers of our two mass spectrometers are detected by multi-channel scaler cards (MCS, 65536 channels, Ortec). The MCS card sorts these events according to time into a selected number of channels of width $t_{\text {dwell }}$ from the beginning of the trigger signal. The statistical error of the counting events $N$ is $\sqrt{N}$. The signal $S$ is computed from $S=N / \Delta t$ with $\Delta t=m_{\text {cycle }} \times t_{\text {dwell }}$ and has the unit counts per second (cps). In the same manner the error of $S$ is obtained $(\Delta S=\sqrt{N} / \Delta t)$. The time resolution $t_{d w e l l}$ per channel applied in the measurements was chosen between $0.1 \mathrm{~ms}$ and $0.4 \mathrm{~ms}$.

\section{B. Mass spectrometer setups}

The plasma is mainly generated in the assumed cylinder with the volume $V=\pi r_{e l}^{2} l_{e l}$ between the stainless steel electrode and the quartz window ${ }^{15}$. An energy-resolving mass spectrometer ${ }^{13}$ (plasma monitor, PM) is used to measure the relative radical density in the plasma volume. The PM is incorporated into the upper, grounded electrode in the radial center of the plasma chamber. In addition, a molecular-beam mass spectrometer (MBMS) is used to measure the radical density on the radial side walls of the plasma chamber. The MBMS is located about $200 \mathrm{~mm}$ from the discharge center in the central plane (at $l=0 \mathrm{~mm}$ ). A detailed description of the PM and the MBMS can be found in Refs. ${ }^{13,16}$ and $^{13,17,18}$, respectively.

The PM is twofold differentially pumped resulting in a background pressure in the plasma monitor vacuum section of the order of $10^{-6} \mathrm{~Pa}$ for a working pressure of $1 \mathrm{~Pa}$ in the plasma chamber. Particles are sampled through two aligned orifices 100 and $500 \mu \mathrm{m}$ in diameter, respectively, and is detected by a secondary electron multiplier (SEM). The PM is here used in the mode for measuring neutrals only. The entrance lens of the PM is set to a voltage of $+100 \mathrm{~V}$ so that plasma ions cannot reach the detector.

The MBMS samples particles at the side wall of the plasma chamber through a carbon tube with an inner diameter of $600 \mu \mathrm{m}$ and a length of $12 \mathrm{~mm}$. The background pressure in the mass spectrometer chamber is of the order of $10^{-6} \mathrm{~Pa}$ for a working pressure of $1 \mathrm{~Pa}$ in the plasma chamber. In addition, the mass spectrometer chamber is equipped with a stainless steel shutter behind the orifice to allow distinguishing the isotropic background signal coming from the background pressure from the signal due to the molecular beam that is formed by the tube. The particle density in this molecular beam is proportional to the particle density in the vesse ${ }^{19}$. The beam-to-background ratio $R$ is mass-dependent and yields for $\mathrm{N}_{2} R_{\mathrm{N}_{2}}=4.4 \pm 0.3$ and for $\mathrm{H}_{2} R_{\mathrm{H}_{2}}=1.5 \pm 0.1$. When the MBMS is used to measure atomic nitrogen (see Sect. IV) no significant signal is detected if the stainless steel shutter blocks the radical beam. That means, the signal of atomic nitrogen has no measurable background but consists only of the collimated beam. 


\begin{tabular}{c|ccc}
$p$ & $B_{e}\left(\mathrm{~cm}^{-1}\right)$ & $\alpha\left(\mathrm{cm}^{-1}\right)$ & $D_{e}\left(\mathrm{~cm}^{-1}\right)$ \\
\hline$X^{1} \Sigma_{g}^{+}$ & 1.998 & 0.0173 & 0.00000573 \\
$B^{3} \Pi_{g}$ & 1.63772 & 0.01816 & 0.00000584 \\
$C^{3} \Pi_{u}$ & 1.82677 & 0.024 & 0.00000511
\end{tabular}

TABLE I. Used molecular constants of $\mathrm{N}_{2}$ for various electronic states $p$ (taken from Ref. ${ }^{28}$ ).

\section{ROTATIONAL TEMPERATURE OF $\mathrm{N}_{2}$}

\section{A. Calculation of the spectrum of $\mathbf{N}_{2}\left(V_{02}^{S P S}\right)$}

For modeling the wall loss it is important to have knowledge of the gas temperature. Therefore, a method for determining the gas temperature in low-temperature nitrogen plasmas will be presented in the following. A frequently used method is the measurement of the rotational temperature of the excited state $C^{3} \Pi_{u}$ of the $\mathrm{N}_{2}$ molecule ${ }^{20-25}$. In the following the correlation between $T_{\text {rot }}^{\mathrm{N}_{2}}$ and $T_{g}$ as well as the determination of $T_{\text {rot }}^{\mathrm{N}_{2}}$ from the line intensity of the rotational spectrum will be presented. The translational energy of $\mathrm{N}_{2}$ molecules is determined by collisions with gas molecules as well as with the plasma-surrounding wall. A fast thermalization between translation and rotation occurs due to collisions so that $T_{\text {rot }}^{\mathrm{N}_{2}} \approx T_{g}{ }^{22-24,26}$. By electron impact excitation of the ground state, the rotational population distribution is directly transferred to the excited state since the electron is too light to change the rotational momentum. A more detailed discussion of the conditions of this method can be found in Biloiu et al. ${ }^{26}$. In the present work the electronic transition of $C^{3} \Pi_{u} \rightarrow B^{3} \Pi_{g}$ of the $\mathrm{N}_{2}$ spectrum of the second positive system (SPS) is considered. From this the established vibrational transition $v^{*}=0 \rightarrow v^{*}=2$ is used ${ }^{22,23}$ which is hereafter abbreviated with $V_{02}^{S P S}$. The line intensity $\dot{N}_{p^{*} v^{*} v^{*} J^{*}}^{p^{*}}$ in the unit of photons per volume and time is the product of the transition probability $A_{p^{\prime c} v^{*} J^{*} J^{*}}^{p^{*}}$ from state $p^{6} v^{6} J^{6}$ into state $p^{\text {" }} v^{\text {" }} J^{\text {" }}$ and the population density $n^{p^{c} v^{c} J^{\natural}}$ of the state $p^{6} v^{6} J^{c}$. In a vibrational transition of an electronic transition, the line intensities $\dot{N}_{J "}^{J^{\prime}}$ of the rotational bands of the considered transition is a function of the following variables ${ }^{23,25,27}$ :

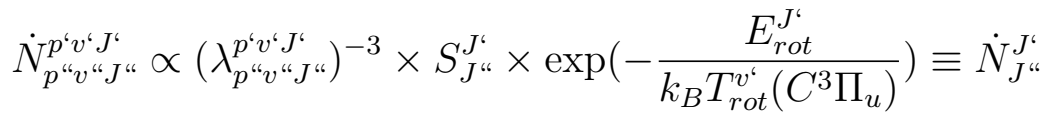

$\left(\lambda_{p^{*} v^{\natural} J^{\natural} J^{\star c}}^{p^{*}}\right.$ - wavelength of the transition, $E_{\text {rot }}^{J^{\star}}$ - rotational energy, $T_{\text {rot }}$ - rotational temperature, $S_{J^{\prime \prime}}^{J^{\star}}$ - Hönl-London factor). The rotational energy is expressed as follows:

$$
E_{\text {rot }}^{J^{\natural}}=B_{v} J^{\bullet}\left(J^{\natural}+1\right)-D_{e} J^{\llcorner 2}\left(J^{\triangleleft}+1\right)^{2},
$$

and

$$
B_{v}=B_{e}-\alpha(v+1 / 2) .
$$

$B_{e}, \alpha$, and $D_{e}$ are molecular constants for the different electronic states. Their used values are given in Table I. 
For the considered transition the coupling of rotation and electronic motion is according to Hund's case $(\mathrm{a})^{29}$. The Hönl-London factors are taken from Ref. ${ }^{25}$ and are expressed for the $\mathrm{P}\left(\Delta J=J^{\natural}-J^{*}=-1\right), \mathrm{Q}(\Delta J=0)$, and $\mathrm{R}(\Delta J=1)$ branch as follows:

$$
\begin{aligned}
& S_{J^{\star}=J^{\star}+1}^{J^{\natural}}=6\left(J^{\natural}+1\right)-10 /\left(J^{\natural}+1\right) \\
& S_{J^{\star}=J^{\natural}}^{J^{\natural}}=10 / J^{\natural}+10 /\left(J^{\natural}+1\right) \\
& S_{J^{\star}=J^{\natural}-1}^{J^{\natural}}=6 J^{\natural}-10 / J^{\natural} .
\end{aligned}
$$

In Eq. 1 it can be seen that with the exception of molecular constants the only variable of $\dot{N}_{J \text { " is }}^{J^{\prime}} T_{r o t}^{\mathrm{N}_{2}}$. Therefore, a spectrum can be calculated and fitted to the measured spectrum by the variation of $T_{\text {rot }}^{\mathrm{N}_{2}}$.

In practise, line broadening caused by the optical spectrometer adds another fitting parameter. In the following, a method is described based on Biloiu et al. ${ }^{26}$ which takes this line broadening into account. For the mathematical description of this line broadening a Gaussian function is used:

$$
f\left(w, \lambda_{x}, \lambda_{0_{J, \Delta J}}\right)=\frac{\sqrt{4 \ln 2}}{\sqrt{\pi} w} \exp \left[-\frac{4 \ln 2}{w^{2}}\left(\lambda_{x}-\lambda_{0, J^{\prime}}^{J^{\star}}\right)^{2}\right]
$$

$\lambda_{x}$ is the spectrometer wavelength which is set by the measuring channels of the CCD array. $\lambda_{0, J^{\prime}}^{J^{\star}}$ denotes the theoretical wavelength position of the observed rotational line, respectively. $w$ is the line width (full width at half maximum - FWHM). The intensities of the P, Q, and $\mathrm{R}$ branch for the quantum numbers $J^{\star}=0-70$ are calculated. To compare the calculated intensities $\dot{N}_{x}^{c}$ with the measured intensities $\dot{N}_{x}^{m}$, all the contributions of the individual intensities at a wavelength $\lambda_{x}$ have to be considered:

$$
\dot{N}_{x}^{c}\left(\lambda_{x}\right)=\sum_{P, Q, R} \sum_{J=1^{\star}}^{70} f\left(w, \lambda_{x}, \lambda_{0, J^{\prime}}^{J^{\star}}\right) \times \dot{N}_{J^{\prime \prime}}^{J^{\star}}\left(\lambda_{0, J^{\prime}}^{J^{\star}}, T_{\text {rot }}^{\mathrm{N}_{2}}\right) .
$$

The calculated and measured spectra are normalized to the respective maximum intensity $\dot{N}_{\text {max }}$. In the experimental spectrum the wavelength scale is shifted such that the maxima of the calculated and the measured spectrum match. The spectra normalized to their respective maxima are compared and $\chi^{2}$ is calculated:

$$
\chi^{2}\left(T_{r o t}^{\mathrm{N}_{2}}, w\right)=\sum_{x=1}^{Z}\left[\frac{\dot{N}_{x}^{m} / \dot{N}_{m a x}^{m}-\dot{N}_{x}^{c} / \dot{N}_{m a x}^{c}\left(T_{r o t}^{\mathrm{N}_{2}}, w\right)}{\Delta\left(\dot{N}_{x}^{m} / \dot{N}_{\max }^{m}\right)}\right]^{2}
$$

$\left(\dot{N}_{x}^{m} / \dot{N}_{m a x}^{m}\right.$ - relative line intensity of the measured spectrum, $\Delta\left(\dot{N}_{x}^{m} / \dot{N}_{\max }^{m}\right)$ - corresponding error of the measured spectrum, $\left(\Delta\left(\dot{N}_{x}^{m} / \dot{N}_{\max }^{m}\right) /\left(\dot{N}_{x}^{m} / \dot{N}_{\max }^{m}\right)=5 \%\right.$ which was estimated by repeating the measurement series once), $\dot{N}_{x}^{c} / \dot{N}_{\text {max }}^{c}$ - relative line intensity of the calculated spectrum, $x$ - index of selected wavelength in the spectrometer, $Z$ - number of considered wavelength channels of the measured spectrum). The rotational temperature of the measured spectrum is determined by minimizing $\chi^{2}$ through variation of $T_{r o t}^{\mathrm{N}_{2}}$ and $w$ in Eq. 9 . 


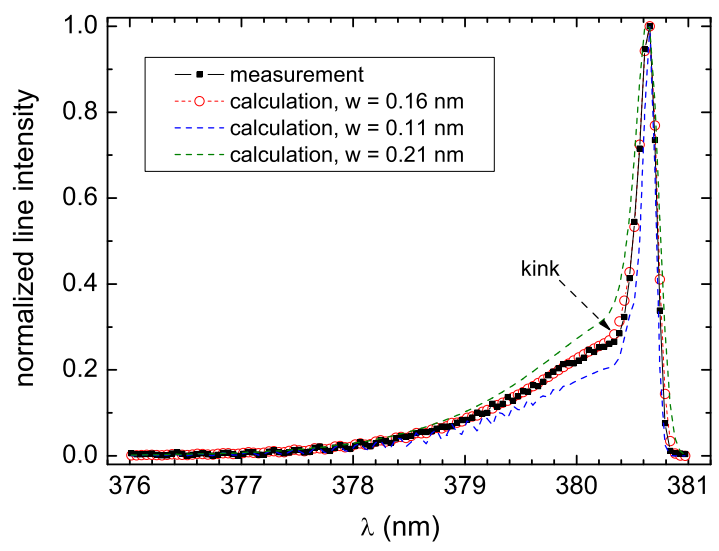

FIG. 2. Comparison between measured and calculated spectrum of the transition $v^{6}=0 \rightarrow v^{\prime \prime}=2$ of the second positive system $\left(C^{3} \Pi_{u} \rightarrow B^{3} \Pi_{g}\right)$ of $\mathrm{N}_{2}$. The plasma parameters are $p=1.5 \mathrm{~Pa}, P=$ $200 \mathrm{~W}$, and $f_{\mathrm{N}_{2}}=56 \%$. The parameters of the simulation where $\chi^{2}$ is minimal are $w=0.16 \mathrm{~nm}$ and $T_{r o t}^{\mathrm{N}_{2}}=480 \mathrm{~K}$. In addition, calculated spectra for line widths of $w=0.11 \mathrm{~nm}$ and $w=0.21 \mathrm{~nm}$ $\left(\left(T_{\text {rot }}^{\mathrm{N}_{2}}=480 \mathrm{~K}\right)\right.$ are plotted.

For the vibrational transition $v^{6}=0 \rightarrow v^{\prime \prime}=2$ the wavelength range between $377.24 \mathrm{~nm}$ and $380.75 \mathrm{~nm}$ with $Z=78$ is considered. For the calculation of the spectrum, the rotational temperature in the excited state $T_{\text {rot }}^{v^{6}}\left(C^{3} \Pi_{u}\right)$ is important (see also Eq. 1). However, for the processes in the plasma the rotational temperature in the ground state is decisive. The rotational population distribution of the ground state is related to the rotational population distribution of the excited state as:

$$
T_{\text {rot }}^{v=0}\left(X^{1} \Sigma_{g}^{+}\right)=\frac{B_{v=0}\left(X^{1} \Sigma_{g}^{+}\right)}{B_{v^{\natural}=0}\left(C^{3} \Pi_{u}\right)} T_{r o t}^{v^{c}=0}\left(C^{3} \Pi_{u}\right) .
$$

Here, $B_{v=0}\left(X^{1} \Sigma_{g}^{+}\right)$and $B_{v^{\prime}=0}\left(C^{3} \Pi_{u}\right)$ denote the rotational constants of the lowest vibrational state of the ground state and from $V_{02}^{S P S}$, respectively. In the present work a $B_{v=0}\left(X^{1} \Sigma_{g}^{+}\right) / B_{v^{\prime}=0}\left(C^{3} \Pi_{u}\right)$ of 1.10 is used which is calculated from data of Ref. ${ }^{28}$. In the following $T_{\text {rot }}^{\mathrm{N}_{2}}$ is always given for the ground state of $\mathrm{N}_{2}$.

The error of the rotational temperature $\Delta T_{\text {rot }}^{\mathrm{N}_{2}}$ is $30 \mathrm{~K}$ (see Refs. ${ }^{20,22}$ ).

\section{B. Comparison with a measured spectrum of $\mathbf{N}_{2}\left(V_{02}^{S P S}\right)$}

In Fig. 2 a measured spectrum for $V_{02}^{S P S}$ of $\mathrm{N}_{2}$ is shown together with the corresponding calculated spectrum. The measurement was performed in a $\mathrm{H}_{2}-\mathrm{N}_{2}$-Ar-Plasma at $f_{\mathrm{N}_{2}}=56 \%$, $p=1.5 \mathrm{~Pa}$, and $P=200 \mathrm{~W}$. The spectrum consists of a peak with a maximum at $\lambda_{x}=$ $380.66 \mathrm{~nm}$, a kink at $\lambda_{x}=380.4 \mathrm{~nm}$ and a tail to lower wavelengths. The calculated spectrum has a minimal $\chi^{2}$ (see Eq. 9) for a $T_{\text {rot }}^{\mathrm{N}_{2}}$ of $480 \mathrm{~K}$ and a $w$ of $0.16 \mathrm{~nm}\left(\chi_{\min }^{2}=9.2 \times 10^{-7}\right)$.

A variation in $T_{r o t}^{\mathrm{N}_{2}}$ varies the height of the tail of the spectrum but leaves the width of 
the peak at $\lambda_{x}=380.66 \mathrm{~nm}$ unchanged. A variation in $w$ varies the height and the shape of the tail as well as the width of the peak at $\lambda_{x}=380.66 \mathrm{~nm}$. The wavelength range from about 379 to $380.4 \mathrm{~nm}$ is very well fitted for $w=0.16 \mathrm{~nm}$. For larger or smaller values of $w$ the agreement between data and model is significantly worse. For comparison, model data for $w=0.11$ and $0.21 \mathrm{~nm}$ are also shown in Fig. 2 .

To cross-check if the line width $w=0.16 \mathrm{~nm}$ determined by the fit is appropriate, $w$ was additionally determined by another method suggested by Biloiu et al. ${ }^{26}$ in which the width of the atomic Ar I line at $415.86 \mathrm{~nm}$ is measured. Based on this method, a value for $w$ of $0.11 \pm 0.01 \mathrm{~nm}$ was determined in the present work. This value is significantly below the value of $0.16 \mathrm{~nm}$ obtained from $\chi^{2}$ minimization. To confirm that $w=0.16 \mathrm{~nm}$ is the optimal value for the spectrometer resolution, the shape of the spectrum will be further discussed. For this purpose, spectra are calculated with a smaller and a larger value of $w$. These curves are presented as dashed lines in Fig. 2. For the first case with $w=0.11 \mathrm{~nm}$ the tail (at $\lambda_{x}<380.35 \mathrm{~nm}$ ) shows an oscillatory structure between $377.0 \lesssim \lambda_{x} \lesssim 379.5 \mathrm{~nm}$, i.e., at high $J^{\prime}$. This oscillatory structure is not observed in the measured spectrum. The absence of the oscillatory structure suggests an actually lower resolution of the spectrometer than $w=0.11 \mathrm{~nm}$. In the other extreme case for $w=0.21 \mathrm{~nm}$ in the range of $379.0 \lesssim$ $\lambda_{x} \lesssim 380.5 \mathrm{~nm}$ the curve shape of the calculated spectrum deviates from the experimental spectrum. Summarizing, a difference in $w=0.16 \mathrm{~nm}$ of $\pm 0.05 \mathrm{~nm}$ leads to a significant deviation of the shape of the calculated spectrum and thus to a higher $\chi^{2}$. It is therefore concluded that $w=0.16 \mathrm{~nm}$ determined by $\chi^{2}$ minimization is the correct parameter.

The spectrum of Fig. 2 was measured in a plasma with a gas mixture of $\mathrm{H}_{2}$ and $\mathrm{N}_{2}$. The plasma parameters were $p=1.5 \mathrm{~Pa}, P=200 \mathrm{~W}$, and $f_{\mathrm{N}_{2}}=56 \%$. Therefore, it is possible to compare $T_{\text {rot }}^{\mathrm{N}_{2}}$ with the rotational temperature $T_{\text {rot }}^{\mathrm{H}_{2}}$ of $\mathrm{H}_{2}$. The method for $T_{\text {rot }}^{\mathrm{H}_{2}}$ was described in Ref. ${ }^{13}$. $T_{\text {rot }}^{\mathrm{H}_{2}}$ was derived from the rotational lines $Q_{1}-Q_{3}$ of the Q-branch of the $\mathrm{H}_{2}$ Fulcher- $\alpha$ diagonal band $\left(v^{6}=v^{*}=2\right)$ with an experimental uncertainty of $50 \mathrm{~K}^{13}$. For the plasma conditions of Fig. 2 a value of $T_{r o t}^{\mathrm{H}_{2}}=(490 \pm 50) \mathrm{K}$ is derived which is within the experimental uncertainty identical with $T_{r o t}^{\mathrm{N}_{2}}=(480 \pm 30) \mathrm{K}$. In Ref. ${ }^{21}$ agreement between $T_{\text {rot }}^{\mathrm{N}_{2}}$ and $T_{\text {rot }}^{\mathrm{H}_{2}}$ was observed, too.

\section{IONIZATION THRESHOLD MASS SPECTROMETRY}

Ionization threshold mass spectrometry (ITMS) is used to measure the relative $\mathrm{N}$ atom density in the afterglow of $\mathrm{N}_{2}$ plasmas as function of time to determine the wall loss time experimentally.

\section{A. ITMS principle}

In the ion source of a mass spectrometer the incoming neutral particles are ionized by electron impact. The thus produced ions are separated according to their mass-to-charge ratio in the quadrupol and finally detected by the SEM. In general, the signal $I$ of a mass spectrometer is proportional to the energy-dependent cross section $\sigma\left(E_{e, i z}\right)$ of the electron- 
collision reaction and the density $n$ of the particles in the ionization chamber (see, e.g. Refs. $\left.{ }^{30,31}\right)$ :

$$
I \propto \sigma\left(E_{e, i z}\right) \times n
$$

where $E_{e, i z}$ is the electron energy of the electrons in the ionizer (subscript " $i z "$ ). Typical ionization energies in the ionizer are $70 \mathrm{eV}$ where the cross section is maximum for most species. At such electron energies different electron-induced reactions are possible: direct ionization of a species, dissociative ionization of molecular species, or even multiple ionization. For example, when measuring $\mathrm{N}$ radicals which result from the parent molecule $\mathrm{N}_{2}$ two processes can contribute to the signal on the mass channel of N. On the one hand, the direct ionization:

$$
\mathrm{N}+e^{-} \rightarrow \mathrm{N}^{+}+2 e^{-}
$$

on the other hand, the dissociative ionization:

$$
\mathrm{N}_{2}+e^{-} \rightarrow \mathrm{N}^{+}+\mathrm{N}+2 e^{-}
$$

Applying Eq. 11 to estimate the intensity ratio of $\mathrm{N}^{+}$originating from $\mathrm{N}\left(I_{\mathrm{N} \rightarrow \mathrm{N}^{+}}\right.$, Eq. 12) to $\mathrm{N}^{+}$originating from $\mathrm{N}_{2}\left(I_{\mathrm{N}_{2} \rightarrow \mathrm{N}^{+}}\right.$, Eq. 13) in a $\mathrm{N}_{2}$ plasma gives the expression $I_{\mathrm{N} \rightarrow \mathrm{N}^{+}} / I_{\mathrm{N}_{2} \rightarrow \mathrm{N}^{+}}=\sigma_{\mathrm{N} \rightarrow \mathrm{N}^{+}} / \sigma_{\mathrm{N}_{2} \rightarrow \mathrm{N}^{+}} \times n_{\mathrm{N}} / n_{\mathrm{N}_{2}}$. For $E_{e, i z}=70 \mathrm{eV}, \sigma_{\mathrm{N} \mathrm{N}^{+}}=1.3 \times 10^{-20} \mathrm{~m}^{2}$ $\left(\right.$ Ref. $^{32}$ ) and $\sigma_{\mathrm{N}_{2} \rightarrow \mathrm{N}^{+}}=0.5 \times 10^{-20} \mathrm{~m}^{2}$ (Ref. ${ }^{33}$ ) are the cross section values for the direct ionization of atomic nitrogen and dissociative ionization of molecular nitrogen, respectively. For a typical dissociation degree of $n_{\mathrm{N}} / n_{\mathrm{N}_{2}}=0.002$ (see Ref. ${ }^{16}$ ) this results in an intensity ratio of $I_{\mathrm{N} \rightarrow \mathrm{N}^{+}} / I_{\mathrm{N}_{2} \rightarrow \mathrm{N}^{+}} \approx 0.005$ which means that for a $\mathrm{N}_{2}$ plasma the mass spectrometer signal at $E_{e, i z}=70 \mathrm{eV}$ on mass channel $14 \mathrm{amu} / \mathrm{e}$ nearly exclusively originates from the $\mathrm{N}_{2}$ background gas and only 0.005 of the signal comes from direct ionization of N. Therefore, at $70 \mathrm{eV}$ quantitative detection of radical particles from the plasma is practically impossible. ITMS exploits the fact that the energy threshold for reaction 13 is larger than that for reaction 12. The energies for other possible pathways are summarized in Table II. To suppress a signal contribution from the dissociative ionization the electron energy $E_{e, i z}$ in the mass spectrometer has to be below the energy threshold of reaction 13. The signal intensity in the range of the threshold energies is small because the cross section of electron-induced ionization is very low.

Because the extraction potential of the ion optics of a mass spectrometer in most cases penetrates into the ionizing volume of the ion source the real electron energy in the extraction volume often differs from the set electron energy $E_{e, i z}$ which is determined by the applied ion source cathode voltage $V_{\text {cath }}$. This results in an ion-source-dependent shift of the ionization energy $\left(e \times \Delta V_{\text {cath }}\right.$, see also Ref. $\left.{ }^{37}\right)$. The electron energy $E_{e, i z}$ is then given by:

$$
E_{e, i z}=e \times\left(V_{\text {cath }}+\Delta V_{\text {cath }}\right)
$$

$E_{e, i z}$ was calibrated by comparing the measured ionization threshold of various stable species such as nitrogen, hydrogen, ammonia, and methane, with their literature values (for nitrogen, see Table II). For both instruments, PM and MBMS, a shift $\Delta V_{\text {cath }}$ of $-2 \mathrm{~V}$ was obtained and is taken into account in the following. 


\begin{tabular}{lll} 
Reaction & $E(\mathrm{eV})$ & Source \\
\hline $\mathrm{e}^{-}+\mathrm{N} \rightarrow \mathrm{N}^{+}+2 \mathrm{e}^{-}$ & 14.5 & 34 \\
$\mathrm{e}^{-}+\mathrm{N} \rightarrow \mathrm{N}(D)+\mathrm{e}^{-}$ & 2.4 & 35 \\
$\mathrm{e}^{-}+\mathrm{N} \rightarrow \mathrm{N}(P)+\mathrm{e}^{-}$ & 3.6 & 35 \\
$\mathrm{e}^{-}+\mathrm{N}_{2} \rightarrow \mathrm{N}^{+}+\mathrm{N}+2 \mathrm{e}^{-}$ & 24.3 & 34 \\
$\mathrm{e}^{-}+\mathrm{N}_{2} \rightarrow \mathrm{N}_{2}^{+}+2 \mathrm{e}^{-}$ & 15.6 & 36 \\
$\mathrm{e}^{-}+\mathrm{N}_{2} \rightarrow \mathrm{N}_{2}(v=10)+\mathrm{e}^{-}$ & 2.7 & 36 \\
$\mathrm{e}^{-}+\mathrm{N}_{2} \rightarrow \mathrm{N}_{2}(\mathrm{~A})+\mathrm{e}^{-}$ & 6.2 & 34 \\
$\mathrm{e}^{-}+\mathrm{N}_{2} \rightarrow 2 \mathrm{~N}+\mathrm{e}^{-}$ & 9.8 & 36
\end{tabular}

TABLE II. Threshold energies $E$ for ionization, excitation and dissociation of atomic and molecular nitrogen.

In contrast to unreactive gas particles radical particles may get lost by contact with surfaces due to their reactivity. Therefore, it is crucial for a quantitative radical detection system that the particle beam from the plasma has no contact with chamber wall elements on the way to the ionizer. This is ensured in the case of the PM and MBMS (see Sec. II) where both electron-impact ionizers are in line of sight to the plasma volume.

\section{B. Identification of atomic nitrogen}

Fig. 3 shows for the PM the signal variation on mass channel $14 \mathrm{amu} / \mathrm{e}$ as function of $E_{e, i z}$ from 12 to $33 \mathrm{eV}$. To measure the thresholds for relevant conditions the plasma off phase was not measured for cold $\mathrm{N}_{2}$ gas but in the afterglow of a pulsed plasma. The signals shown are the steady state values at the end of the plasma on or plasma off phase, respectively. Each data point was acquired by summarizing many pulses for a fixed value of $E_{e, i z}$ with the MCS card. The plasma parameters for the discharge in pure $\mathrm{N}_{2}$ shown in Fig. 3 are $p=3 \mathrm{~Pa}$, a rf power of $P=300 \mathrm{~W}, t_{\text {on }}=10 \mathrm{~ms}, t_{\text {off }}=10 \mathrm{~ms}$, and $t_{\text {dwell }}=1 \mathrm{~ms}$. With such a variation of $E_{e, i z}$ it is possible to distinguish the $\mathrm{N}^{+}$ions originating from atomic $\mathrm{N}$ from $\mathrm{N}^{+}$produced from molecular $\mathrm{N}_{2}$.

In Fig. 3 the PM signals for $P_{o n}$ and $P_{\text {off }}$ are clearly different in the energy range of $12 \leq E_{e, i z} \leq 25 \mathrm{eV}$. In this energy range the PM signal during $P_{\text {on }}$ is about one order of magnitude higher than that during $P_{o f f}$. For $E_{e, i z}>25 \mathrm{eV}$ the PM signals of $P_{\text {on }}$ and $P_{\text {off }}$ approach each other. Both PM signals are characterized by edges where the slope changes significantly. In Fig. 3 these edges are located at around 14 and $25 \mathrm{eV}$.

The PM signal in the electron energy region of $12 \leq E_{e, i z} \leq 25 \mathrm{eV}$ during $P_{o n}$ is assigned to atomic nitrogen from the plasma since only during $P_{\text {on }}$ an explicit PM signal is measured. The PM signal for $E_{e, i z}>25 \mathrm{eV}$ originates predominantly from molecular nitrogen which is abound in both phases. Furthermore, the measured edges coincide within the experimental uncertainty with the threshold energies for direct $\left(E_{t h r, 1}\left(\mathrm{~N} \rightarrow \mathrm{N}^{+}\right)=14.5 \mathrm{eV}\right.$, Eq. 12) and dissociative ionization $\left(E_{t h r, 2}\left(\mathrm{~N}_{2} \rightarrow \mathrm{N}^{+}\right)=24.3 \mathrm{eV}\right.$, Eq. 13).

A significant decrease of the PM signal with decreasing energy occurs in the range of $12 \leq E_{e, i z} \leq 15 \mathrm{eV}$. One would expect that below $E_{t h r, 1}\left(\mathrm{~N} \rightarrow \mathrm{N}^{+}\right)=14.5 \mathrm{eV}$ no significant 


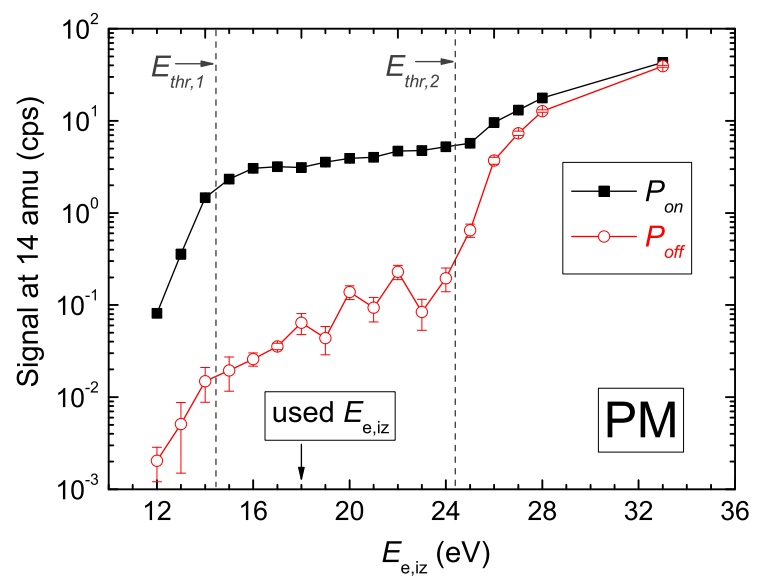

FIG. 3. Ionization threshold mass spectrometry of $\mathrm{N}^{+}$on mass channel $14 \mathrm{amu} / \mathrm{e}$ during plasmaon $\left(P_{o n}\right)$ and plasma-off $\left(P_{o f f}\right)$ state in a $\mathrm{N}_{2}$ discharge at a pressure of $3 \mathrm{~Pa}$ and a rf power of $300 \mathrm{~W}$ (pulsed mode: $10 \mathrm{~ms} P_{o n}$ and $10 \mathrm{~ms} P_{o f f}$ ) as function of the electron energy $E_{e, i z}$ of the ionizer measured with the plasma monitor $(\mathrm{PM})$. The threshold energies $E_{t h r, 1}=14.5 \mathrm{eV}$ and $E_{t h r, 2}=24.3 \mathrm{eV}$ of the direct ionization of $\mathrm{N}$ and dissociative ionization of $\mathrm{N}_{2}$ from Table II are shown.

PM signal would be detected. But the lowest electron energy where still a signal is measured is $E_{e, i z}=12 \mathrm{eV}$. This should be due to the fact that $\mathrm{N}^{+}$is not only produced by direct ionization of ground state $\mathrm{N}\left({ }^{4} S\right)$, but also by direct ionization of the metastable states $\mathrm{N}\left({ }^{2} D\right)$ and $\mathrm{N}\left({ }^{2} P\right)$ which have ionization energies of 12.1 and $10.7 \mathrm{eV}$, respectively (see Table II). The $\mathrm{N}\left({ }^{2} D\right)$ threshold of $E_{t h r}\left(\mathrm{~N}^{2} D \rightarrow \mathrm{N}^{+}\right)=12.1 \mathrm{eV}$ agrees with the observed value of $E_{e, i z}=12 \mathrm{eV}$. Therefore, we attribute the PM signal in the range of $12 \leq E_{e, i z} \leq 15 \mathrm{eV}$ to the metastable state $\mathrm{N}\left({ }^{2} D\right)$.

For the measurement of the decay of atomic nitrogen with time in pulsed plasmas a fixed electron energy $E_{e, i z}$ is used. With knowledge of Fig. 3 for the mass channel $14 \mathrm{amu} / \mathrm{e}$ a $E_{e, i z}$ of $18 \mathrm{eV}$ is selected for the measurements with the PM (see Sec. V).

In Fig. 4 equivalent measurements with the MBMS for the same plasma conditions are shown. Qualitatively, the behavior of the MBMS measurements is the same as in the case of the PM. A quantitative difference between them is that in the MBMS measurement the energy of the edge between $12 \leq E_{e, i z} \leq 18 \mathrm{eV}$ in Fig. 4 is at $(16 \pm 1) \mathrm{eV}$ whereas for the PM measurement it is at $(14 \pm 1) \mathrm{eV}$. It is assumed that this lower edge of the PM measurement is due to $\mathrm{N}\left({ }^{2} D\right)$. Therefore, it is concluded that at the plasma chamber side walls were the MBMS is located the density of $\mathrm{N}\left({ }^{2} D\right)$ is negligible. For the measurement of the relative radical density in pulsed plasmas (see Sec. V) a fixed electron energy of $E_{e, i z}=18 \mathrm{eV}$ is used for the MBMS.

In Figs. 3 and 4 it can be seen that although there is a substantial difference between the $P_{o n}$ and $P_{o f f}$ signals also in the $P_{o f f}$ state the signal during $P_{o f f}$ is significant. However, even without plasma for pure $\mathrm{N}_{2}$ gas in the chamber signals at $14 \mathrm{amu} / \mathrm{e}$ and $E_{e, i z}=12 \mathrm{eV}$ 


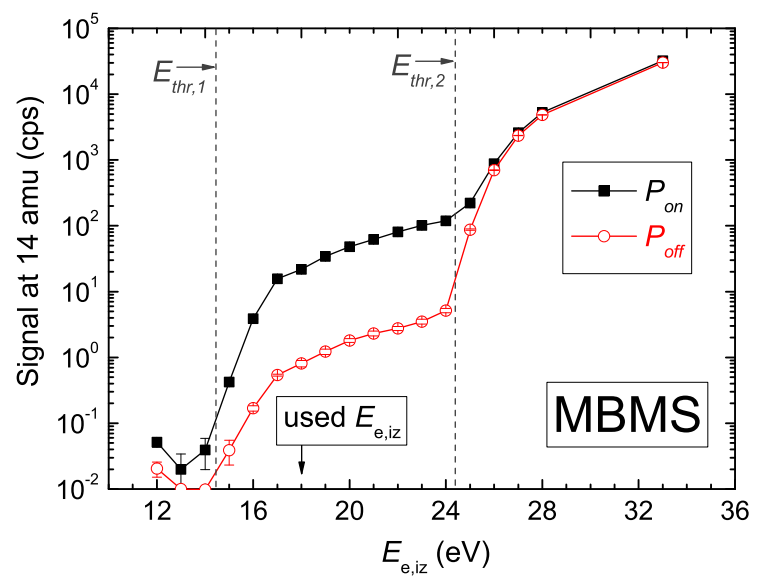

FIG. 4. Same as in Fig. 3 measured with the molecular-beam mass spectrometer (MBMS).

are detected (average signal rate of 0.1 counts/s for the PM and 0.01 counts/s for the MBMS in steady state). These signals are attributed to dissociation of $\mathrm{N}_{2}$ at the hot filament in the ion source of the mass spectrometers. The $\mathrm{N}_{2}$ originates from the isotropic background gas in the mass spectrometer chambers. Such a dissociation of the parent molecule was also observed by Kae-Nune et al. ${ }^{38}$ in ITMS measurements of atomic hydrogen.

So far it was assumed for the ITMS technique that molecular nitrogen only occurs in the ground state. However, during plasma operation $\mathrm{N}_{2}$ can also occur in vibrationallyexcited levels $\mathrm{N}_{2}(v i b)$ or as metastable $\mathrm{N}_{2}(A)$ which denotes the $A^{3}{ }^{3}{ }_{u}^{+}$state of $\mathrm{N}_{2}$. In the following, the influence of vibrationally excited and metastable states which have lower threshold energy for $\mathrm{N}^{+}$production than ground state $\mathrm{N}_{2}$, is discussed. For the chosen electron energy of $E_{e, i z}=18 \mathrm{eV}$ excited $\mathrm{N}_{2}$ molecules can be ionized if their potential energy is at least $24.3 \mathrm{eV}-18 \mathrm{eV}=6.3 \mathrm{eV}$ above the ground state of $\mathrm{N}_{2}(X)$. An energy of $6.3 \mathrm{eV}$ above the ground state corresponds to a vibrational level of about $v=26$. The vibrational population can be estimated with a typical vibrational temperature of $T_{v i b}=11,000 \mathrm{~K}$ (see Ref. $\left.{ }^{39}\right)$ to $\mathrm{N}_{2}(v i b) / \mathrm{N}_{2}(X) \propto \exp (-6.3 \mathrm{eV} / 1 \mathrm{eV})=0.002$. For a vibrational level higher than $6.3 \mathrm{eV}(v>26)$ the relative density is even lower than 0.002. For the cross section for the dissociative ionization of the vibrational levels $(v>26)$ we estimate that $\sigma_{\mathrm{N}_{2}(v i b) \rightarrow \mathrm{N}^{+}}$ is of the order of $\sigma_{\mathrm{N} \rightarrow \mathrm{N}^{+}}$. Using Eq. 11 we summarize that the contribution of $\mathrm{N}_{2}($ vib) to the $\mathrm{N}^{+}$signal can be neglected for our conditions. The metastable state $\mathrm{N}_{2}(A)$ has a density ratio $\mathrm{N}_{2}(A) / \mathrm{N}_{2}(X)$ in the order of $\mathrm{N} / \mathrm{N}_{2}(X)$ as shown by a rate equation mode ${ }^{16}$. The excitation energy of $\mathrm{N}_{2}(A)$ is $6.3 \mathrm{eV}$. However, right at the threshold the cross section $\sigma_{\mathrm{N}_{2}(A) \rightarrow \mathrm{N}^{+}}\left(E_{e, i z}=18 \mathrm{eV}\right)$ goes to 0 and, hence, the influence of $\mathrm{N}_{2}(A)$ on the $\mathrm{N}^{+}$signal can also be neglected.

The linearity of the intensity scales of the secondary electron multipliers (SEM) of the mass spectrometers is a further prerequisite for the measurement of the decay time. The linearity was checked by two methods. First, the isotopic ratio of neon was measured and compared to the theoretical isotopic ratio. A description of the procedure can be found 


\begin{tabular}{cccc|ccc}
$\begin{array}{c}p \\
(\mathrm{~Pa})\end{array}$ & $\begin{array}{c}P_{r f} \\
(\mathrm{~W})\end{array}$ & $\begin{array}{c}t_{\text {on }} \\
(\mathrm{ms})\end{array}$ & $\begin{array}{c}t_{\text {off }} \\
(\mathrm{ms})\end{array}$ & $\begin{array}{c}n_{e} \\
\left(10^{16} \mathrm{~m}^{-3}\right)\end{array}$ & $\begin{array}{c}T_{e} \\
(\mathrm{eV})\end{array}$ & $\begin{array}{c}T_{\text {rot }}^{\mathrm{N}_{2}} \\
(\mathrm{~K})\end{array}$ \\
\hline 3.0 & 300 & 2 & 20 & 3.6 & 2.7 & 560 \\
5.0 & 250 & 3 & 30 & 3.8 & 2.3 & 590 \\
7.5 & 220 & 5 & 50 & 3.7 & 2.1 & 620 \\
10.0 & 205 & 10 & 100 & 3.6 & 2.0 & 660
\end{tabular}

TABLE III. Used plasma conditions ( $p$ - total pressure, $P_{r f}$ - rf power, $t_{o n}$ - duration of plasma-on phase, $t_{o f f}$ - duration of plasma-off phase) and measurement results $\left(n_{e}\right.$ - electron density, $T_{e}$ electron temperature, $T_{r o t}^{\mathrm{N}_{2}}$ - rotational temperature of $\mathrm{N}_{2}$ ) of the considered $\mathrm{N}_{2}$ plasma. $n_{e}, T_{e}$ were measured with the Langmuir probe and $T_{r o t}^{\mathrm{N}_{2}}$ with OES in steady-state plasmas.

in Ref. ${ }^{13}$. Both measured and theoretical ratios agree within the experimental uncertainty. Second, the decay of the Ar signal is measured with both the PM and the MBMS after stopping the gas supply of Ar into the plasma chamber. The experiment was carried out for two different initial Ar pressures. Both measuring devices show that the signal decay is exponential as function of the time over an intensity range of more than 4 orders of magnitude. Likewise, both measuring devices yield the same decay time. Based on these experimental results we conclude that both instruments the PM as well as the MBMS have a linear intensity scale in the range of interest here.

\section{RESULTS}

Measurements were carried out in pure $\mathrm{N}_{2}$ plasmas for pressures of 3.0, 5.0, 7.5, and $10.0 \mathrm{~Pa}$. The plasma parameters $n_{e}$ and $T_{e}$ determined with the Langmuir probe and $T_{\text {rot }}^{\mathrm{N}_{2}}$ determined by OES are shown in Table III together with the plasma conditions. The rf power was adjusted such that the variation of the electron density with increasing pressure is minimized. $n_{e}$ was kept at $n_{e}=(3.7 \pm 0.1) \times 10^{16} \mathrm{~m}^{-3}$. As expected, $T_{e}$ decreases with increasing pressure from 2.7 to $2.0 \mathrm{eV}$. $T_{\text {rot }}^{\mathrm{N}_{2}}$ increases with increasing pressure from $560 \mathrm{~K}$ at $3.0 \mathrm{~Pa}$ to $660 \mathrm{~K}$ at $10.0 \mathrm{~Pa}$ and thus varies by $100 \mathrm{~K}$. The mean value of $T_{\text {rot }}^{\mathrm{N}_{2}}$ is $610 \mathrm{~K}$ which will be used as gas temperature $T_{g}$ in the following for all pressures.

The internal plasma parameters $n_{e}, T_{e}$, and $T_{\text {rot }}^{\mathrm{N}_{2}}$ listed in Table III were measured in steady-state plasmas without pulsing. On a time scale of $\lesssim 100 \mu$ s after plasma ignition the electron temperature and electron density reach steady state ${ }^{40-42}$. Therefore, it is assumed that the measured $T_{e}$ and $n_{e}$ of the steady state plasmas are also valid for the pulsed plasmas for $t>100 \mu \mathrm{s}$. The plasma electrons take a key position in the heating mechanism of the gas species ${ }^{13,23}$ (e.g. heating by electron-induced dissociation of the stable molecular gas species) resulting in a certain gas temperature. It is assumed that once $T_{e}$ and $n_{e}$ are in steady state, also the rotational temperature is in steady state.

Nitrogen radicals are produced by electron-induced dissociation of $\mathrm{N}_{2}$ and are lost by recombination at the wall. In this work, the temporal afterglow of the $P_{\text {off }}$ phase for times larger than $100 \mu \mathrm{s}$ is considered. Within a time scale of less than $10 \mu \mathrm{s}$ the electron temperature decreases to less than $1 \mathrm{eV}$ (see Refs. ${ }^{40,41}$ ) so that plasma electrons are no 
longer able to dissociate or ionize gas species. Therefore, the afterglow measurements of the present work show only the loss process of the radicals.

As discussed in Sec. IV the mass spectrometers PM and MBMS operate on the mass channel $14 \mathrm{amu} / \mathrm{e}$ using an electron energy of $E_{e, i z}=18 \mathrm{eV}$ to measure the relative $\mathrm{N}$ atom density in neutral gas mode during the pulsed $\mathrm{N}_{2}$ plasma. The duration times $t_{\text {on }}$ and $t_{\text {off }}$ of the $P_{\text {on }}$ and $P_{\text {off }}$ phases for different pressures were chosen such that the decay process can be measured optimally. The duty cycle $t_{o n} /\left(t_{o n}+t_{o f f}\right)$ is constant for all studied pressures. The used $t_{\text {on }}$ and $t_{\text {off }}$ are included in Table III. The signals of the PM for the different investigated pressures are shown in Fig. 5 as a function of time $t$. In Fig. 5(a) the signal (absolute number of counts) is shown for a full pulse at a pressure of $3 \mathrm{~Pa}$. For this measurement $3.8 \times 10^{5}$ full cycles were accumulated. With a dwell time of $10^{-4} \mathrm{~s}$ and a number of channels of 220 this corresponds to a total measurement time of more than $2 \mathrm{~h}$. The $P_{\text {on }}$ phase is characterized by a fast signal increase within the first $0.8 \mathrm{~ms}$. Then the signal saturates and remains constant for the remaining $P_{\text {on }}$ time. The absolute number of counts is about 600 resulting in good counting statistics (counting error $\sim 4 \%$ ). In the $P_{\text {off }}$ phase, the signal falls off rapidly within the first $4 \mathrm{~ms}$. For larger times $t$ in the $P_{\text {off }}$ phase the signal exhibits a small background. For all studied pressures the background signal of the PM is within the experimental uncertainty constant at about $0.2 \mathrm{cps}$. The $\mathrm{x}$-axis (time $t$ ) is chosen such that at $t=0$ the power-off phase starts. In Fig. 5(b-e) the signals for 3.0, 5.0, 7.5, and 10.0 $\mathrm{Pa}$ are shown together with the fit curves (see below). The signals of Fig. 5(b-e) are converted to counts per second. The $\mathrm{x}$ and $\mathrm{y}$ axis in Fig. 5(b-e) are for all plots the same. Therefore, the signals can be compared with each other. For the different considered pressures different $t_{\text {on }}$ and $t_{\text {off }}$ values (see Table III) were chosen. Therefore, the measurement at $3 \mathrm{~Pa}$ starts at $t=-2 \mathrm{~ms}$ and the measurement at $p=10 \mathrm{~Pa}$ at $t=-10 \mathrm{~ms}$. The steady-state signal of the $P_{\text {on }}$ phase decreases with increasing pressure.

In the $P_{\text {off }}$ phase the signal decreases exponentially. The following exponential function is used to fit the data:

$$
y=C e^{\left(-t / t_{w \mathrm{~N}}\right)}+y_{0}
$$

$C$ and $y_{0}$ are fitting constants and $t_{w \mathrm{~N}}$ is the decay time. $y_{0}$ accounts for the signal background for large $t$. For better comparison the normalized $(y(t=0)=1)$ fit curves are shown together in one plot in Fig. 5(f). It can be clearly seen that with increasing pressure the decay time increases. The such determined decay times are plotted in Fig. 6 as function of $p$. $t_{w \mathrm{~N}}$ increases linearly from 0.31 to $0.82 \mathrm{~ms}$ as $p$ increases from 3.0 to $10.0 \mathrm{~Pa}$. The $t_{w \mathrm{~N}}$ data as function of $p$ are fitted using a linear fit with the fitting constants $A_{w \mathrm{~N}}$ and $B_{w \mathrm{~N}}$ which are the intersection with the $\mathrm{y}$-axis and the slope, respectively. The values are $A_{w \mathrm{~N}}^{\mathrm{PM}}=8.2 \times 10^{-5} \mathrm{~s}$ and $B_{w \mathrm{~N}}^{\mathrm{PM}}=7.6 \times 10^{-5} \mathrm{~s} \mathrm{~Pa}^{-1}$. It has to be mentioned that in Fig. 5(f) the background level cannot be compared with each other for different pressures because these are normalized curves with different background-to-maximum ratios.

The data measured by MBMS at the sidewall of the plasma chamber are shown in Fig. 7. These data are measured simultaneously with the PM data shown in Fig. 5. Qualitatively, the results are similar to the PM measurements of Fig. 5. However, the signal peak is much broader. The signal during $P_{\text {on }}$ just reaches a maximum. The decay of the MBMS signals in the afterglow is also fitted according to Eq. 15. Due to the fact that the signals as function 

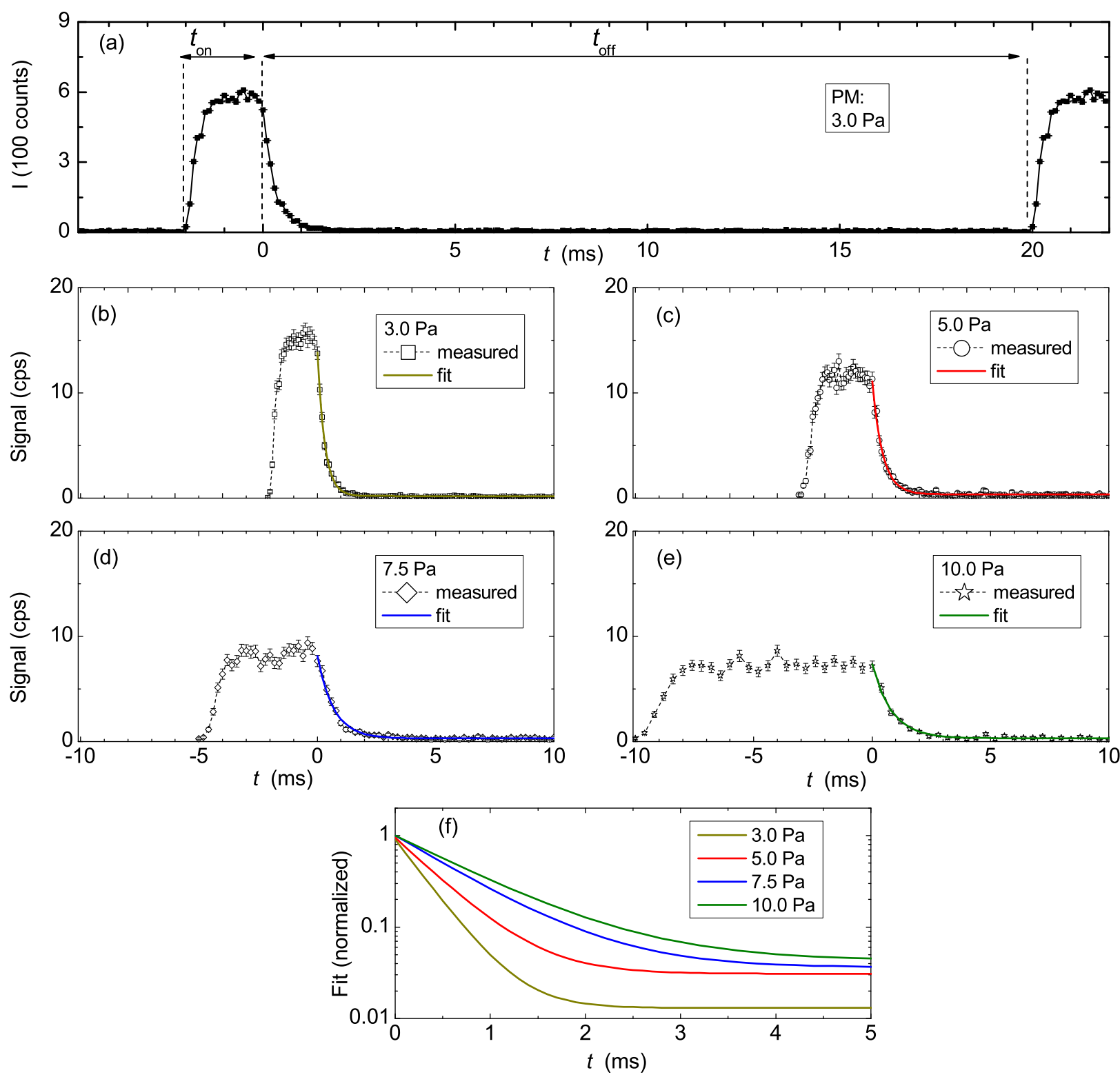

FIG. 5. Signal at mass channel $14 \mathrm{amu} / \mathrm{e}$ and an electron energy of $18 \mathrm{eV}$ measured with the plasma monitor (PM) in a pulsed $\mathrm{N}_{2}$ plasma for different pressures as function of time $t$. (a) shows the signal for a complete pulse cycle consisting of the power-on phase with time $t_{\text {on }}$ and power-off phase with time $t_{\text {off }}$. Measurements and corresponding fit curves are shown for the pressures 3.0 $\mathrm{Pa}(\mathrm{b}), 5.0 \mathrm{~Pa}(\mathrm{c}), 7.5 \mathrm{~Pa}(\mathrm{~d})$, and $10.0 \mathrm{~Pa}(\mathrm{e})$. The $\mathrm{x}$-axis is chosen such that at $t=0$ the power-off phase starts. In Fig. f normalized fit curves are shown for comparison. 


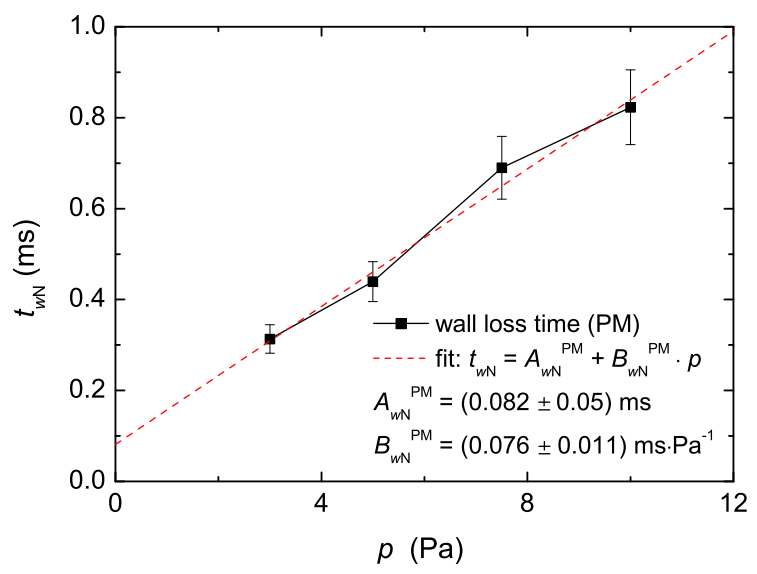

FIG. 6. Wall loss time $t_{w \mathrm{~N}}$ of atomic nitrogen from afterglow measurements in a $\mathrm{N}_{2}$ plasma as function of the pressure $p$ measured with the plasma monitor PM (black squares). A fit of $t_{w \mathrm{~N}}$ as a function of $p$ is shown as dashed curve.

of $t$ do neither show a clear saturation nor a distinct start of the decay at $y(t=0)$, the fit was applied for slight higher values of $t>0(0<t<0.1 \mathrm{~ms}$ for 3.0 to $7.5 \mathrm{~Pa}, 1.6 \mathrm{~ms}$ for 10.0 Pa measurement) where a decay can be observed clearly (see Figs. 7(b-e)). For the same pressure the decay times measured with the MBMS are longer than these of the PM. Similar to the PM measurements they increase with increasing pressure. These decay times are plotted in Fig. 8 as function of $p . t_{w \mathrm{~N}}$ increases linearly from 1.4 to $3.8 \mathrm{~ms}$ as $p$ increases from 3.0 to $10.0 \mathrm{~Pa}$. A linear fit for $t_{w \mathrm{~N}}$ as function of $p$ yields $A_{w \mathrm{~N}}^{\mathrm{MBMS}}=5.5 \times 10^{-4} \mathrm{~s}$ and $B_{w \mathrm{~N}}^{\mathrm{MBMS}}=2.7 \times 10^{-4} \mathrm{~s} \mathrm{~Pa}^{-1}$. The most important difference between the MBMS and the PM measurement is that $t_{w \mathrm{~N}}$ measured with the MBMS is considerably larger than that of the PM measurement. Furthermore, the slopes are different. The slope ratio is $B_{w \mathrm{~N}}^{\mathrm{MBMS}} / B_{w \mathrm{~N}}^{\mathrm{PM}}=3.6 \pm 0.9$. To clarify this difference, a model of the wall loss time will be discussed in the following section.

\section{DISCUSSION}

\section{A. Theoretical description of the wall loss time}

In $\mathrm{N}_{2}$ plasmas the $\mathrm{N}$ atoms are produced almost exclusively from the electron-induced dissociation of $\mathrm{N}_{2}$ with the corresponding rate coefficient $K_{\text {diss }}$ and are lost to the wall. The wall loss is determined by diffusion and a surface loss part where $\mathrm{N}$ atoms recombine to form $\mathrm{N}_{2}$. Other loss processes such as three-body recombination in the plasma volume or pumping can be neglected for the present conditions ${ }^{3,4}$. The wall loss time $t_{w \mathrm{~N}}$, which is the inverse of the wall loss rate coefficient, is the mean time for a nitrogen atom to get lost at the plasma surrounding wall. In a zero dimensional ansatz the $\mathrm{N}$ atom density is given by 

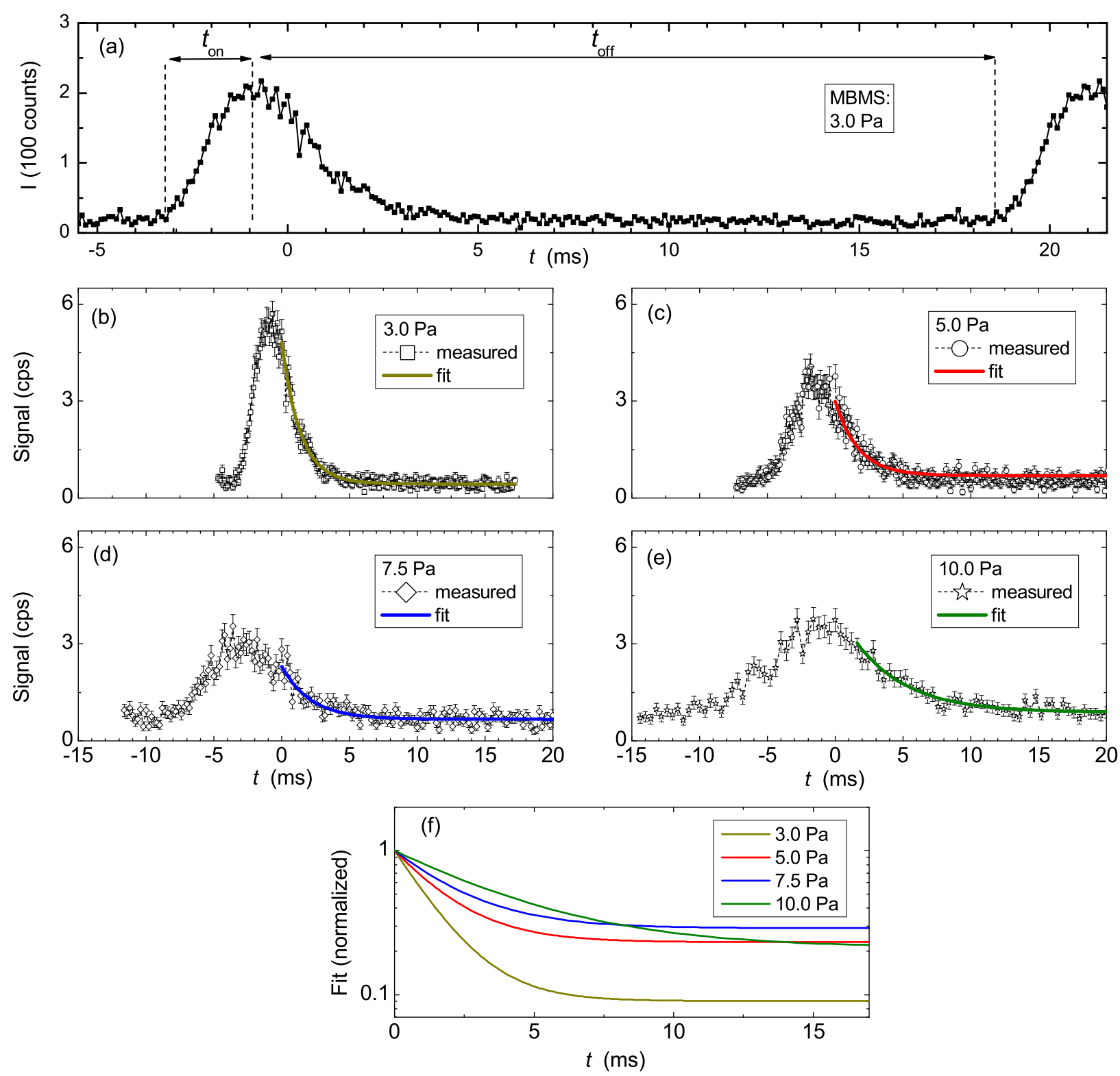

FIG. 7. Signal at mass channel $14 \mathrm{amu} / \mathrm{e}$ and an electron energy of $18 \mathrm{eV}$ measured with the molecular-beam mass spectrometer (MBMS) in a pulsed $\mathrm{N}_{2}$ plasma for different pressures as function of time $t$. (a) shows the signal for a complete pulse cycle consisting of the power-on phase with time $t_{o n}$ and power-off phase with time $t_{o f f}$. Measurements and corresponding fit curves are shown for the pressures 3.0 $\mathrm{Pa}(\mathrm{b}), 5.0 \mathrm{~Pa}(\mathrm{c}), 7.5 \mathrm{~Pa}(\mathrm{~d})$, and 10.0 $\mathrm{Pa}(\mathrm{e})$. The $\mathrm{x}$-axis is chosen such that at $t=0$ the power-off phase starts. In Fig. f normalized fit curves are shown for comparison. 


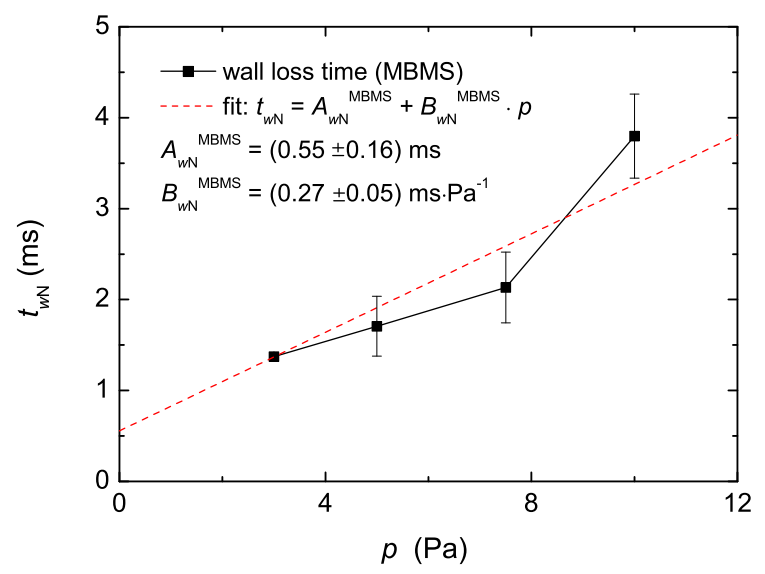

FIG. 8. Wall loss time $t_{w \mathrm{~N}}$ of atomic nitrogen from afterglow measurements in a $\mathrm{N}_{2}$ plasma as function of the pressure $p$ measured with the molecular-beam mass spectrometer MBMS (black squares). A fit of $t_{w \mathrm{~N}}$ as a function of $p$ is drawn as dashed curve.

the continuity equation:

$$
\frac{\partial n_{\mathrm{N}}}{\partial t}=2 n_{\mathrm{N}_{2}} \times n_{e} \times K_{\text {diss }}\left(T_{e}\right)-\frac{n_{\mathrm{N}}}{t_{w \mathrm{~N}}}
$$

In the plasma afterglow $\mathrm{N}$ atoms are no longer generated from the electron-induced dissociation of $\mathrm{N}_{2}$ since the energy of the electrons drops sharply within a few $\mu$ s after switching off the rf power ${ }^{40,41}$. The loss process, though, takes place in the millisecond range. Therefore, in the afterglow Eq. 16 simplifies to:

$$
\frac{\partial n_{\mathrm{N}}}{\partial t}=-\frac{n_{\mathrm{N}}}{t_{w \mathrm{~N}}}
$$

If $t_{w \mathrm{~N}}$ is constant as function of $t$ the solution of the differential Eq. 17 is an exponential decay of the $\mathrm{N}$ density:

$$
n_{\mathrm{N}}=n_{\mathrm{N}, 0} \times e^{-t / t_{w \mathrm{~N}}}
$$

with $n_{\mathrm{N}, 0}$ being the $\mathrm{N}$ density at $t=0$, i.e., the $\mathrm{N}$ density at the time when plasma is switched off. The observed decay of the measured atomic nitrogen signals in the $P_{\text {off }}$ phase is exponential (see Sec. V). Based on this, we assign the experimentally-obtained decay time of the afterglow measurements to the wall loss time. In general, $t_{w \mathrm{~N}}$ can be described by a diffusive part $t_{D \mathrm{~N}}$ and a surface loss part $t_{s \mathrm{~N}}{ }^{43,44}$ :

$$
t_{w \mathrm{~N}}=t_{s \mathrm{~N}}+t_{D \mathrm{~N}}
$$


The surface loss time is given by ${ }^{15,43}$ :

$$
t_{s \mathrm{~N}}=\frac{V}{A} \frac{2\left(2-\beta_{\mathrm{N}}\right)}{\beta_{\mathrm{N}}} \frac{1}{v_{\mathrm{N}}}
$$

( $V$ - plasma volume, $A$ - surface area where $\mathrm{N}$ can be lost, $\beta_{\mathrm{N}}$ - surface loss probability for $\mathrm{N}, v_{\mathrm{N}}=\sqrt{8 k_{B} T_{\mathrm{N}} / \pi M_{\mathrm{N}}}-$ mean velocity of $\mathrm{N}, M_{\mathrm{N}}$ - mass of $\mathrm{N}, T_{\mathrm{N}}$ - temperature of N). The diffusion time of atomic nitrogen is given by ${ }^{15,43}$ :

$$
t_{D \mathrm{~N}}=\frac{\Lambda^{2}}{D_{\mathrm{N}}}=\frac{\Lambda^{2} \times p}{\tilde{D}_{\mathrm{N}}}
$$

with $\Lambda$ being the diffusion length, $D_{\mathrm{N}}$ the diffusion constant, and $\tilde{D}_{\mathrm{N}}$ the pressureindependent diffusion constant. Since $t_{w \mathrm{~N}}$ is a linear function of $p$ it is more convenient to rewrite Eq. 19 as a function of $p$ :

$$
t_{w \mathrm{~N}}=A_{w \mathrm{~N}}+B_{w \mathrm{~N}} \times p
$$

with

$$
A_{w \mathrm{~N}}=t_{s \mathrm{~N}}
$$

and

$$
B_{w \mathrm{~N}}=\frac{\Lambda^{2}}{\tilde{D}_{\mathrm{N}}} .
$$

The intersection $A_{w \mathrm{~N}}$ of $t_{w \mathrm{~N}}$ with the $\mathrm{y}$-axis and the slope $B_{w \mathrm{~N}}$ were determined experimentally in Sec. $\mathrm{V}$ from the measurements of $t_{w \mathrm{~N}}$ for different pressures. According to Eqs. 23 and $24 A_{w \mathrm{~N}}$ and $B_{w \mathrm{~N}}$ are identified with the surface loss time and the diffusive part, respectively. As can be seen in Figs. 6 and 8, $A_{w \mathrm{~N}}$ has a rather low contribution on the measured $t_{w \mathrm{~N}}$. For example, for the $t_{w \mathrm{~N}}$ measurement with the PM presented in Fig. $6 A_{w \mathrm{~N}}$ contributes at the lowest considered pressure of $3 \mathrm{~Pa}$ with $26 \%$ to $t_{w \mathrm{~N}}$. For higher pressures the contribution is even lower. Therefore, for our case $t_{w \mathrm{~N}}$ is dominated by diffusion, i.e., $t_{w \mathrm{~N}} \approx t_{D \mathrm{~N}}$.

For the diffusion constant several models ${ }^{16,45}$ exist depending on the intermolecular potential for the collision of $\mathrm{N}$ with $\mathrm{N}_{2}$. Furthermore, the diffusion constant is a function of the particle temperature. The temperature of the $\mathrm{N}$ atom is, however, a priori unknown. Here we assume that the $\mathrm{N}$ temperature is equal to the background gas temperature for the considered plasma conditions ${ }^{8}$.

With the chosen zero-dimensional ansatz $t_{w \mathrm{~N}}$ is a function of the geometry of the plasma chamber. In this respect, the volume to surface area ratio $V / A$ is an important parameter for the description of $t_{s \mathrm{~N}}$ (see Eq. 20) and the diffusion length $\Lambda$ for $t_{D \mathrm{~N}}$ (see Eq. 21). In our experimental setup the geometry of the plasma chamber does not have a simple cylindrical geometry and the plasma-surrounding walls consist of different materials (see Fig. 1) which have different $\beta_{\mathrm{N}}$ (see Eq. 20). Therefore, a simple cylindrical geometry cannot be applied and further assumptions have to be made. Furthermore, the positions of the mass spectrometers are important. The PM measures the $\mathrm{N}$ density at the radial center 
of the plasma volume $(r=0 \mathrm{~mm}$ and $l=30 \mathrm{~mm})$ whereas the MBMS measures at the radial side walls $(r=200 \mathrm{~mm}$ and $l=0 \mathrm{~mm})$. Therefore, the relevant geometry has to be discussed individually for both setups.

The following considerations shall illustrate that the different wall loss times measured with the MBMS and PM can be attributed to the different positions of the mass spectrometers in the plasma chamber which leads to a different effective geometry. In doing this, the ratio $B_{w \mathrm{~N}}^{M B M S} / B_{w \mathrm{~N}}^{P M}$ of the slopes determined from the linear fits in Figs. 6 and 8 will be estimated based on a model for $t_{w \mathrm{~N}}$. According to Eq. 24 the ratio of the slopes is independent of the diffusion constant. $B_{w \mathrm{~N}}^{M B S} / B_{w \mathrm{~N}}^{P M}$ is, therefore, equal to the ratio of the square of the diffusion lengths $\left(\Lambda^{\mathrm{MBMS}} / \Lambda^{\mathrm{PM}}\right)^{2}$. For cylindrical geometry $\Lambda$ is given by ${ }^{43}$ :

$$
\frac{1}{\Lambda^{2}}=\left(\frac{\pi}{l}\right)^{2}+\left(\frac{2.405}{r}\right)^{2}
$$

Now, the effective $\Lambda$ has to be discussed for both PM and MBMS. For the PM a cylinder without radial side wall at $r=r_{e l}$ between the upper electrode and the quartz window is considered as the relevant volume. Because there is no radial side wall at $r_{e l}$ and due to an aspect ratio $l_{e l} / r_{e l} \lesssim 1$ only axial losses to the upper stainless steel electrode or lower quartz dome are taken into account. This is equivalent to $r \rightarrow \infty$ (see Ref. ${ }^{15}$ ) such that the second term in Eq. 25 vanishes. In our experiment the plasma interacts with two chamber wall materials: stainless steel (electrode) and quartz (dielectric window). Quartz has a significantly lower $\beta_{\mathrm{N}}$ than stainless steel (see Ref. ${ }^{46}$ ). Therefore, it is assumed that atomic nitrogen is lost only at the stainless steel electrode ${ }^{15}$. For the PM the diffusion length is obtained by solving the diffusion equation for a 1-dimensional case where the radical is lost on the one and reflected on the other electrode surface. The result is $\Lambda^{\mathrm{PM}}=l_{e l} / \sqrt{3}=35 \mathrm{~mm}$ (for details, see Ref. ${ }^{16}$ ). For the MBMS the geometry is rather complex. The MBMS measures at the radial side walls of the chamber. As simplest approach a cylinder with $l=l_{c h}=360 \mathrm{~mm}$ and $r=200 \mathrm{~mm}$ which is the distance between plasma center and MBMS entrance orifice is assumed. Since $l_{c h}>r=200 \mathrm{~mm}$ Eq. 25 can be simplified to $\Lambda^{\mathrm{MBMS}} \approx r / 2.405=83 \mathrm{~mm}$. Under these assumptions, the ratio of the slopes is:

$$
B_{w \mathrm{~N}}^{M B M S} / B_{w \mathrm{~N}}^{P M}=\left(\Lambda^{\mathrm{MBMS}} / \Lambda^{\mathrm{PM}}\right)^{2} \approx\left(\frac{r}{2.405} \times \frac{\sqrt{3}}{l_{e l}}\right)^{2}=5.8
$$

For comparison, the experimentally obtained slope ratio of Sec. $\mathrm{V}$ is $B_{w \mathrm{~N}}^{\mathrm{MBMS}} / B_{w \mathrm{~N}}^{\mathrm{PM}}=3.6 \pm 0.9$. It has to be noted that $\Lambda^{\mathrm{MBMS}}=83 \mathrm{~mm}$ is only a very rough upper estimate. Considering Eq. 25 and noting that $l$ is finite and a significant loss of radicals occurs at the steel electrode, $\Lambda^{\text {MBMS }}$ should be substantially smaller than $83 \mathrm{~mm}$ resulting in a lower slope ratio. Despite the large inherent uncertainties, Eq. 26 yields a rough estimate of the experimentally obtained slope ratio. Summarizing, the difference of wall loss times measured with MBMS and PM is attributed to the different diffusion lengths.

As final result, we will derive the surface loss probability $\beta_{\mathrm{N}}$ of atomic nitrogen on stainless steel from the wall loss time data of the PM. For this, the intersection $A_{w \mathrm{~N}}$ of $t_{w \mathrm{~N}}$ with the y-axis of Fig. 6 is analyzed applying Eqs. 20 and 23. For the analysis the volume to 
surface ratio and the radical temperature have to be known. The volume of the assumed cylinder is $V=\pi r_{e l}^{2} l_{e l}$. As mentioned above the atomic nitrogen radical is lost only at the area of the upper electrode, i.e., $A=\pi r_{e l}^{2}$ (see Ref. ${ }^{15}$ ). Therefore, the volume to surface ratio is $V / A=l_{e l}$. As also discussed above, the radical temperature is assumed to be equivalent to the gas temperature $\left(T_{\mathrm{N}}=T_{g}\right)$. With the experimentally obtained value of $A_{w \mathrm{~N}}^{\mathrm{PM}}=(8.2 \pm 5) \times 10^{-5} \mathrm{~s}$, Eqs. 20 and 23 , and the assumptions for $V / A$ and $T_{\mathrm{N}}$ a $\beta_{\mathrm{N}}$ of $1.2 \pm 0.3$ is derived. A surface loss probability of higher than 1 is not reasonable. Therefore, it is concluded that the surface loss probability of atomic nitrogen on stainless steel lies in the range of 0.9 to 1.0 for the conditions of our experiment.

Due to the larger scatter of the MBMS data resulting in a much larger uncertainty of $A_{w \mathrm{~N}}^{\mathrm{MBMS}}$, we cannot derive a $\beta_{\mathrm{N}}$ from these data. Nevertheless, we can infer a large $\beta_{\mathrm{N}}$ from the MBMS data because as mentioned in Sec. II B no significant $N$ signal is detected when the stainless steel shutter blocks the beam inside the MBMS chamber. For low $\beta_{\mathrm{N}} \mathrm{N}$ should survive many wall collisions and reach the ionizer. This is not the case. Therefore, we conclude that $\beta_{\mathrm{N}}$ measured with the MBMS is expected to be comparably large as for the PM measurements.

\section{B. Comparison with literature}

Takashima et al. ${ }^{5}$ determined $t_{w \mathrm{~N}}$ in the afterglow of a pulsed capacitively-coupled $\mathrm{N}_{2}$ plasma with vacuum ultraviolet absorption spectroscopy (VUVAS) in the pressure range from 1.3 to $27 \mathrm{~Pa}$. The measured $t_{w \mathrm{~N}}$ corresponds to the wall loss time. $t_{w \mathrm{~N}}$ increases linearly from 10 to $20 \mathrm{~ms}$ as $p$ increases from 1.3 to $13 \mathrm{~Pa}$. Hence, $t_{w \mathrm{~N}}$ is proportional to the pressure which is in agreement with the results of the present work. Takashima et al. used the same model for the description of $t_{w \mathrm{~N}}$ as in the present work (see Sec. IV). They determined a $\beta_{\mathrm{N}}$ of 0.03 for $\mathrm{N}$ on stainless steel for an atomic nitrogen temperature of $400 \mathrm{~K}$ which is a factor of 30 smaller than the one determined here.

Kang et al. ${ }^{6}$ investigated experimentally the $\mathrm{N}$ atom density in an pulsed inductively coupled $\mathrm{N}_{2}$ plasma as a function of time in the afterglow with two-photon absorption laserinduced fluorescence (TALIF). The absolute $\mathrm{N}$ atom density in the afterglow was shown for 2.7 and $27 \mathrm{~Pa}$ and exhibits a simple exponential decrease with time. The decay times were determined for pressures between 2.7 and $1000 \mathrm{~Pa}$. The lowest decay time was measured for a pressure of $2.7 \mathrm{~Pa}$ and was $t_{w \mathrm{~N}}=3.3 \mathrm{~ms}$. $t_{w \mathrm{~N}}$ is almost constant up to a pressure of about $40 \mathrm{~Pa}$. For higher pressures $p>40 \mathrm{~Pa} t_{w \mathrm{~N}}$ increased with increasing pressure. Kang et al. used also the same theory for the description of $t_{w \mathrm{~N}}$ as the present work (see Sec. VI). They took the measured $\mathrm{N}_{2}$ rotational temperature of $400 \mathrm{~K}$ as gas temperature and as the $\mathrm{N}$ temperature, too. A $\beta_{\mathrm{N}}$ of 0.02 gave a good agreement between the calculated and the measured $t_{w \mathrm{~N}}$ as a function of $p .2 / 3$ of the plasma chamber wall of Kang et al. consisted of stainless steel and $1 / 3$ of glass. For calculation of $\beta_{\mathrm{N}}$ from $t_{w \mathrm{~N}}$ the whole wall loss area was considered. Therefore, the obtained $\beta_{\mathrm{N}}$ is a mean value for the two different wall materials. The specified $\beta_{\mathrm{N}}$ in Kang et al. is an average over values for various surfaces.

The similarities between Kang et al., Takashima et al., and the present study are that both observed in the afterglow of a pulsed plasma a simple exponential decrease of the $\mathrm{N}$ 
atom density with time and the measured $t_{w \mathrm{~N}}$ is well described by a diffusion model (see Sec. VI). In spite of similarities in discharge geometries, discharge volumes, pressure range, and similar power densities, the measured wall loss times of Refs. ${ }^{5,6}$ are at least one order of magnitude higher than the values of the present work. Because both Refs. ${ }^{5,6}$ apply the same model as in the present work this difference in $t_{w \mathrm{~N}}$ leads also to more than one order of magnitude difference in $\beta_{\mathrm{N}}$. We can only speculate about the reason for this. One possibility could be a different surface temperature. Therefore, we consider the work of Gaboriau et al. ${ }^{10}$.

Gaboriau et al. ${ }^{10}$ determined $\beta_{\mathrm{N}}$ of $\mathrm{N}$ on iron in a glass reactor at $40-400 \mathrm{~Pa}$ in the $P_{\text {off }}$ phase. They determined $\beta_{\mathrm{N}}$ with a fiber optic catalytic probe (see also Ref. ${ }^{11}$ ). They determined $\beta_{\mathrm{N}}$ for various pressures. In the range between $130<p<400 \mathrm{~Pa} \beta_{\mathrm{N}}$ is independent of pressure. In the range between $40<p<130 \mathrm{~Pa} \beta_{\mathrm{N}}$ increases with decreasing $p$. For a surface temperature of $400 \mathrm{~K} \beta_{\mathrm{N}}$ increases from $0.06(130 \mathrm{~Pa})$ to $0.33(40 \mathrm{~Pa})$ but no interpretation of this increase was provided. Furthermore, they studied two different temperatures of the catalytic probe: 310 und $400 \mathrm{~K}$. They observed for $130<p<400 \mathrm{~Pa}$ an increase of $\beta_{\mathrm{N}}$ from $0.02(310 \mathrm{~K})$ to $0.06(400 \mathrm{~K})$ by a factor of 3 and thus showed that $\beta_{\mathrm{N}}$ depends sensitively on the temperature of the surface.

If we assume that the relevant surface temperature is equal to the measured gas temperature we come to the following considerations: In the work of Takashima et al. ${ }^{5}$ and Kang et al. $^{6}$ the gas temperature was $400 \mathrm{~K}$. In contrast, in our experiments the measured gas temperature was $600 \mathrm{~K}$. Accordingly the surface temperature in our experiment is assumed to be $600 \mathrm{~K}$ while it is only $400 \mathrm{~K}$ in the other two references ${ }^{5,6}$. As shown by Gaboriau et al. a higher surface temperature causes a higher $\beta_{\mathrm{N}}$. Therefore, we suppose that the difference between Refs. ${ }^{5,6}$ and the present work is due to the considerable different temperatures.

Another issue is the surface condition of the plasma chambers for the different studies. For the capacitively coupled plasma of Takashima et al. ${ }^{5}$ the quartz window is most probably sputtered and the sputtered material is re-deposited as thin film on the stainless steel surface. For Kang et al. ${ }^{6}$ we can only speculate that capacitive coupling was also present to some extent from his planar coil. At least in the beginning we observed quartz sputtering in our setup using standard matching. To minimize sputtering we have made substantial effort to suppress capacitive coupling of our ICP to avoid ions with high energies ${ }^{13,47}$. In a former publication $^{13}$ we showed that in our inductively coupled plasma the ion energies are of the order of $20 \mathrm{eV}$ and, therefore, sputtering of quartz can be neglected. Furthermore, comparisons between the stainless steel electrode and an exchangeable electrode cover made of stainless steel results in the same surface loss probability of hydrogen in the case of hydrogen plasmas (see Ref. ${ }^{8}$ ). Therefore, it is concluded that the stainless steel surface of our plasma chamber is not modified during plasma operation and that the plasma-surface interaction of the radicals occurs with clean stainless steel.

In this context we want to add the following remark: Also for Pyrex different research groups obtained very different $\beta_{\mathrm{N}}$ values. As can be seen in Fig. 6 of Ref. ${ }^{48}$ published values for $\beta_{\mathrm{N}}$ on Pyrex measured vary by up to two orders of magnitude. Therefore, in future studies more attention has to be paid on critical parameters highlighted in this study such as surface condition (temperature, purity), vessel geometry, and radical temperature. 


\section{SUMMARY}

In the afterglow of an pulsed inductively coupled $\mathrm{N}_{2}$ plasma relative $\mathrm{N}$ atom densities were measured as a function of time by ionization threshold mass spectrometry (ITMS) in order to determine the wall loss time $t_{w \mathrm{~N}}$ from the exponential decay curves. The procedure was performed with two mass spectrometers at different positions in the plasma chamber to study the dependence of $t_{w \mathrm{~N}}$ on the positions in the plasma chamber. The first mass spectrometer (plasma monitor, PM) was located in the radial center of the plasma chamber. The second mass spectrometer (molecular-beam mass spectrometer, MBMS) measured the radical density on the radial side walls of the plasma chamber.

The wall loss times were determined for pressures of 3.0, 5.0, 7.5, and 10 Pa. For this conditions also the internal plasma parameters electron density $n_{e}$ and electron temperature $T_{e}$ determined with a Langmuir probe and the rotational $\mathrm{N}_{2}$ temperature $T_{\text {rot }}^{\mathrm{N}_{2}}$ determined with optical emission spectroscopy were measured. For $T_{\text {rot }}^{\mathrm{N}_{2}}$ a procedure was presented to evaluate the spectrum of the transition $v^{6}=0 \rightarrow v^{*}=2$ of the second positive system $\left(C^{3} \Pi_{u} \rightarrow B^{3} \Pi_{g}\right)$ of $\mathrm{N}_{2}$. With this method a gas temperature of $610 \mathrm{~K}$ was determined. It is assumed that the temperature of atomic nitrogen is equal to the gas temperature.

With the two mass spectrometers it was observed that the wall loss times of atomic nitrogen increase with increasing pressure. The wall loss time measured in the radial center of the plasma chamber increases linearly from $0.31 \mathrm{~ms}$ for $3 \mathrm{~Pa}$ to $0.82 \mathrm{~ms}$ for $10 \mathrm{~Pa}$. The wall loss time measured at the vessel wall is about 4 times larger than that of the PM. A model was applied to describe the measured $t_{w \mathrm{~N}}$. The main loss mechanism of atomic nitrogen for the considered pressure range is diffusion to the wall. The surface loss probability $\beta_{\mathrm{N}}$ of atomic nitrogen on stainless steel was derived from $t_{w \mathrm{~N}}$ and was found to be about unity (0.9-1.0) for the present conditions. With the model it was shown that the difference of wall loss times measured at the two positions can be attributed to the different diffusion lengths.

\section{ACKNOWLEDGMENTS}

We gratefully acknowledge help from T. Dürbeck for technical assistance.

\section{REFERENCES}

${ }^{1}$ S. Lee, S. Bang, S. Jeon, S. Kwon, W. Jeong, S. Kim, and H. Jeon, "Characteristics of hafnium-zirconium-oxide film treated by remote plasma nitridation," Journal of The Electrochemical Society 155, H516-H519 (2008).

${ }^{2}$ C. Hinkle and G. Lucovsky, "Remote plasma-assisted nitridation (RPN): applications to $\mathrm{Zr}$ and Hf silicate alloys and $\mathrm{Al}_{2} \mathrm{O}_{3}$," Applied Surface Science 216, 124 - 132 (2003), Proceedings of the Fourth International Symposium on the Control of Semiconductor Interfaces Karuizawa, Japan, October 21-25, 2002.

${ }^{3}$ E. G. Thorsteinsson and J. T. Gudmundsson, "A global (volume averaged) model of a 
nitrogen discharge: I. steady state," Plasma Sources Science and Technology 18, 045001 (2009).

${ }^{4}$ H. Singh, J. W. Coburn, and D. B. Graves, "Recombination coefficients of $\mathrm{O}$ and N radicals on stainless steel," Journal of Applied Physics 88, 3748-3755 (2000).

${ }^{5}$ S. Takashima, K. Takeda, S. Kato, M. Hiramatsu, and M. Hori, "Surface loss probability of nitrogen atom on stainless-steel in $\mathrm{N}_{2}$ plasma afterglow," Japanese Journal of Applied Physics 49, 076101 (2010).

${ }^{6}$ N. Kang, F. Gaboriau, S.-G. Oh, and A. Ricard, "Modeling and experimental study of molecular nitrogen dissociation in an Ar- $\mathrm{N}_{2}$ ICP discharge," Plasma Sources Science and Technology 20, 045015 (2011).

${ }^{7}$ S. F. Adams and T. A. Miller, "Surface and volume loss of atomic nitrogen in a parallel plate rf discharge reactor," Plasma Sources Science and Technology 9, 248 (2000).

${ }^{8}$ M. Sode, T. Schwarz-Selinger, W. Jacob, and H. Kersten, "Surface loss probability of atomic hydrogen for different electrode cover materials investigated in $\mathrm{H}_{2}$-Ar low-pressure plasmas," Journal of Applied Physics 116, 013302 (2014).

${ }^{9}$ H. Kersten, D. Rohde, J. Berndt, H. Deutsch, and R. Hippler, "Investigations on the energy influx at plasma processes by means of a simple thermal probe," Thin Solid Films 377-378, 585 - 591 (2000), international Conference on Metallurgic Coatings and Thin Films.

${ }^{10}$ F. Gaboriau, U. Cvelbar, M. Mozetič, A. Erradi, and B. Rouffet, "Comparison of TALIF and catalytic probes for the determination of nitrogen atom density in a nitrogen plasma afterglow," Journal of Physics D: Applied Physics 42, 055204 (2009).

${ }^{11}$ M. Mozetič, U. Cvelbar, A. Vesel, A. Ricard, D. Babic, and I. Poberaj, "A diagnostic method for real-time measurements of the density of nitrogen atoms in the postglow of an Ar- $\mathrm{N}_{2}$ discharge using a catalytic probe," Journal of Applied Physics 97, 103308 (2005).

${ }^{12}$ J. Röpcke, P. B. Davies, N. Lang, A. Rousseau, and S. Welzel, "Applications of quantum cascade lasers in plasma diagnostics: a review," Journal of Physics D: Applied Physics 45, 423001 (2012).

${ }^{13}$ M. Sode, T. Schwarz-Selinger, and W. Jacob, "Quantitative determination of massresolved ion densities in $\mathrm{H}_{2}$-Ar inductively coupled radio frequency plasmas," Journal of Applied Physics 113, 093304 (2013).

${ }^{14} \mathrm{~V}$. A. Kadetov, Diagnostics and modeling of an inductively coupled radio frequency discharge in hydrogen, PhD thesis, Ruhr Universität Bochum (2004).

${ }^{15} \mathrm{M}$. Sode, T. Schwarz-Selinger, and W. Jacob, "Ion chemistry in $\mathrm{H}_{2}$-Ar low temperature plasmas," Journal of Applied Physics 114, 063302 (2013).

${ }^{16} \mathrm{M}$. Sode, Quantitative Beschreibung von Wasserstoff-Stickstoff-Argon-Mischplasmen, $\mathrm{PhD}$ thesis (in German), Universität Kiel (2013).

${ }^{17}$ M. Bauer, T. Schwarz-Selinger, H. Kang, and A. von Keudell, "Control of the plasma chemistry of a pulsed inductively coupled methane plasma," Plasma Sources Sci. Technol. 14, 543-548 (2005).

${ }^{18}$ M. Bauer, T. Schwarz-Selinger, W. Jacob, and A. v. Keudell, "Growth precursor for a$\mathrm{C}: \mathrm{H}$ film deposition in pulsed inductively coupled methane plasmascontrol of the plasma chemistry of a pulsed inductively coupled methane plasma," J. Appl. Phys. 98, 073302 
(2005).

${ }^{19} \mathrm{~W}$. Walcher, "Über eine Ionenequelle für massenspektroskopische Isotopentrennung," Zeitschrift für Physik 122, 62-85 (1944).

${ }^{20}$ P. Scheubert, U. Fantz, P. Awakowicz, and H. Paulin, "Experimental and theoretical characterization of an inductively coupled plasma source," J. Appl. Phys. 90, 587 (2001).

${ }^{21}$ U. Fantz, "Emission spectroscopy of molecular low pressure plasmas," Contrib. Plasma Phys. 44, 508-515 (2004).

${ }^{22} \mathrm{~K}$. Behringer, "Diagnostics and modelling of ECRH microwave discharges," Plasma Physics and Controlled Fusion 33, 997 (1991).

${ }^{23}$ V. M. Donnelly and M. V. Malyshev, "Diagnostics of inductively coupled chlorine plasmas: Measurements of the neutral gas temperature," Applied Physics Letters 77, 2467 (2000).

${ }^{24}$ G. P. Davis and R. A. Gottscho, "Measurement of spatially resolved gas-phase plasma temperatures by optical emission and laser-induced fluorescence spectroscopy," Journal of Applied Physics 54, 3080-3086 (1983).

${ }^{25}$ T. Sakamoto, H. Matsuura, and H. Akatsuka, "Spectroscopic study on the vibrational populations of $\mathrm{N}_{2} C^{3} \Pi$ and $B^{3} \Pi$ states in a microwave nitrogen discharge," Journal of Applied Physics 101, 023307 (2007).

${ }^{26}$ C. Biloiu, X. Sun, Z. Harvey, and E. Scime, "An alternative method for gas temperature determination in nitrogen plasmas: Fits of the bands of the first positive system $\left(B^{3} \Pi_{g} \rightarrow\right.$ $\left.A^{3} \Sigma_{u}^{+}\right)$," Journal of Applied Physics 101, 073303 (2007).

${ }^{27}$ S. Saloum, M. Naddaf, and B. Alkhaled, "Diagnostics of $\mathrm{N}_{2}$-Ar plasma mixture excited in a $13.56 \mathrm{MHz}$ hollow cathode discharge system: application to remote plasma treatment of polyamide surface," Journal of Physics D: Applied Physics 41, 045205 (2008).

${ }^{28} \mathrm{~B}$. Krames, Räumliche Konzentrationsverteilungen von $\mathrm{N}_{2}$-Triplett-Zuständen im elektrodennahen Plasma einer RF-Niederdruckentladung, PhD thesis (in German), Technische Universität Chemnitz (1999).

${ }^{29}$ G. Herzberg, Moleklspektren und Moleklstruktur. I.: Zweiatomige Molekle. (Steinkopff, Dresden, 1939).

${ }^{30}$ H. Singh, J. W. Coburn, and D. B. Graves, "Appearance potential mass spectrometry: Discrimination of dissociative ionization products," J. Vac. Sci. Technol. A 18, 299 (2000).

${ }^{31}$ S. Matsuda, H. Shimosato, M. Yumoto, and T. Sakai, "Detection of nitrogen metastable molecules by using the threshold ionization mass spectrometry," Electrical Engineering in Japan 147, 17-24 (2004).

${ }^{32}$ Y.-K. Kim and J.-P. Desclaux, "Ionization of carbon, nitrogen, and oxygen by electron impact," Phys. Rev. A 66, 012708 (2002).

${ }^{33}$ Y. Itikawa, "Cross sections for electron collisions with nitrogen molecules," Journal of Physical and Chemical Reference Data 35, 31-53 (2006).

${ }^{34}$ S. Agarwal, B. Hoex, M. C. M. van de Sanden, D. Maroudas, and E. S. Aydil, "Absolute densities of $\mathrm{N}$ and excited $\mathrm{N}_{2}$ in a $\mathrm{N}_{2}$ plasma," Applied Physics Letters 83, 4918-4920 (2003).

${ }^{35}$ B. Sourd, P. Andr, J. Aubreton, and M.-F. Elchinger, "Influence of the excited states of atomic nitrogen $\mathrm{N}\left({ }^{2} \mathrm{D}\right)$ and $\mathrm{N}\left({ }^{2} \mathrm{P}\right)$ on the transport properties of nitrogen. Part i: Atomic nitrogen properties," Plasma Chemistry and Plasma Processing 27, 35-50 (2007). 
${ }^{36}$ F. R. Gilmore, "Potential energy curves for $\mathrm{N}_{2}, \mathrm{NO}, \mathrm{O}_{2}$ and corresponding ions," Journal of Quantitative Spectroscopy and Radiative Transfer 5, 369 - IN3 (1965).

${ }^{37}$ H. Singh, J. W. Coburn, and D. B. Graves, "Mass spectrometric detection of reactive neutral species: Beam-to-background ratio," J. Vac. Sci. Technol. A 17, 2447-2455 (1999).

${ }^{38} \mathrm{P}$. Kae-Nune, J. Perrin, J. Guillon, and J. Jolly, "Mass spectrometry detection of radicals in $\mathrm{SiH}_{4}-\mathrm{CH}_{4}-\mathrm{H}_{2}$ glow discharge plasmas," Plasma Sources Sci. Technol. 4, 250-259 (1995), modeling ITMS $\mathrm{s}=0.006$.

${ }^{39}$ T. Kimura and H. Kasugai, "Experiments and global model of inductively coupled rf Ar/ $\mathrm{N}_{2}$ discharges," Journal of Applied Physics 108, 033305 (2010).

${ }^{40}$ S. Ashida, M. R. Shim, and M. A. Lieberman, "Measurements of pulsed-power modulated argon plasmas in an inductively coupled plasma source," Journal of Vacuum Science and Technology A: Vacuum, Surfaces, and Films 14, 391-397 (1996).

${ }^{41}$ M. A. Lieberman and S. Ashida, "Global models of pulse-power-modulated high-density, low-pressure discharges," Plasma Sources Sci. Technol. 5, 145 (1996).

${ }^{42}$ M. Osiac, T. Schwarz-Selinger, D. O'Connell, B. Heil, Z. L. Petrovic, M. M. Turner, T. Gans, and U. Czarnetzki, "Plasma boundary sheath in the afterglow of a pulsed inductively coupled RF plasma," Plasma Sources Sci. Technol. 16, 355-363 (2007).

${ }^{43}$ P. J. Chantry, "A simple formula for diffusion calculations involving wall reflection and low density," J. Appl. Phys. 62, 1141-1148 (1987).

${ }^{44}$ M. A. Lieberman and A. J. Lichtenberg, Principles of plasma discharges and materials processing (John Wiley and Sons, Inc., Hoboken, New Jersey, 2005).

${ }^{45}$ G. N. Hays, C. J. Tracy, and H. J. Oskam, "Surface catalytic efficiency of a sputtered molybdenum layer on quartz and Pyrex for the recombination of nitrogen atoms," The Journal of Chemical Physics 60, 2027-2034 (1974).

${ }^{46} \mathrm{~K}$. Kutasi and J. Loureiro, "Role of the wall reactor material on the species density distributions in an $\mathrm{N}_{2} \mathrm{O}_{2}$ post-discharge for plasma sterilization," J. Phys. D. 40, 5612 (2007).

${ }^{47}$ K. Polozhiy and T. Schwarz-Selinger, "Suppresion of capacitive coupling in a pulsed inductively coupled RF discharge," (2011), unpublished.

${ }^{48}$ L. Lefvre, T. Belmonte, and H. Michel, "Modeling of nitrogen atom recombination on Pyrex: Influence of the vibrationally excited $\mathrm{N}_{2}$ molecules on the loss probability of N," Journal of Applied Physics 87, 7497-7507 (2000). 